\title{
Phytochemical-rich herbal formula ATG-125 protects against sucrose-induced gastrocnemius muscle atrophy by rescuing Akt signaling and improving mitochondrial dysfunction in young adult mice
}

\author{
CHING-CHUAN YEH $^{1 *}$, HSUAN-MIAO LIU ${ }^{1 *}$, MING-CHUNG LEE ${ }^{2}$, YANN-LII LEU ${ }^{3,4}$ \\ WEI-HAN CHIANG ${ }^{5}$, HEN-HONG CHANG ${ }^{6}$ and TZUNG-YAN LEE ${ }^{1,7,8}$
}

\begin{abstract}
${ }^{1}$ Graduate Institute of Traditional Chinese Medicine, School of Chinese Medicine, College of Medicine, Chang Gung University, Taoyuan 33302; ${ }^{2}$ Brion Research Institute of Taiwan, New Taipei City 23143; ${ }^{3}$ Graduate Institute of Nature Products, College of Medicine, Chang Gung University, Taoyuan 33302; ${ }^{4}$ Tissue Bank, Chang Gung Memorial Hospital, Taoyuan 33305; ${ }^{5}$ Department of Rehabilitation, Cheng-Hsin General Hospital, Taipei 11283; ${ }^{6}$ Graduate Institute of Integrated Medicine, China Medical University, Taichung 40402;

${ }^{7}$ Graduate Institute of Clinical Medical Sciences, College of Medicine, Chang Gung University, Taoyuan 33302; ${ }^{8}$ Department of Traditional Chinese Medicine, Chang Gung Memorial Hospital, Keelung City 20401, Taiwan, R.O.C.
\end{abstract}

Received August 23, 2021; Accepted November 18, 2021

DOI: $10.3892 / \mathrm{mmr} .2021 .12572$

\begin{abstract}
The antioxidant capability of herbal remedies has attracted widespread attention, but their molecular mechanisms in a muscle atrophy model have not been explored. The aim of the present study was to compare the bioactivity of sucrose challenged mice following treatment with ATG-125. Here, through a combination of transcriptomic and biomedical analysis, herbal formula ATG-125, a phytochemical-rich formula, was identified as a protective factor against muscle atrophy in sucrose challenged mice. Gene ontology (GO) identified differentially expressed genes that were primarily enriched in the 'negative regulation of proteolysis', 'cellular amino acid metabolic process', 'lipoprotein particle' and 'cell cycle', all of which were associated with the ATG-125-mediated prevention of muscle atrophy, particularly with regard to mitochondrial biogenesis. In skeletal muscle, a set of
\end{abstract}

Correspondence to: Professor Tzung-Yan Lee, Graduate Institute of Traditional Chinese Medicine, School of Chinese Medicine, College of Medicine, Chang Gung University, 259 Wen-Hwa 1st Road, Kwei-Shan, Taoyuan 33302, Taiwan, R.O.C.

E-mail: joyamen@mail.cgu.edu.tw

Dr Hen-Hong Chang, Graduate Institute of Integrated Medicine, China Medical University, 91 Hsueh-Shih Road, Taichung 40402, Taiwan, R.O.C.

E-mail: tcmchh55@gmail.com

*Contributed equally

Key words: muscle atrophy, inflammation, mitochondrial dysfunction, transcriptome mitochondrial-related genes, including angiopoietin-like 4, nicotinamide riboside kinase 2 (Nmrk2), pyruvate dehydrogenase lipoamide kinase isozyme 4 , Asc-type amino acid transporter 1 and mitochondrial uncoupling protein 3 (Ucp3) were markedly upregulated following ATG-125 intervention. An increase in Nmrk2 and Ucp3 expression were noted after ATG-125 treatment, in parallel with upregulation of the 'nicotinate and nicotinamide metabolism' pathway, as determined using the Kyoto Encyclopedia of Genes and Genomes (KEGG). Furthermore, KEGG pathway analysis revealed the downregulation of 'complement and coagulation cascades', 'cholesterol metabolism', 'biosynthesis of amino acids' and 'PPAR signaling pathway', which were associated with the downregulation of serine (or cysteine) peptidase inhibitor clade A member (Serpina)3, Serpinalb, Serpinald, Serpinale, apolipoprotein (Apo)al and Apoa2, all of which were cardiovascular and diabetes-associated risk factors and were regulated by ATG-125. In addition, ATG-125 treatment resulted in downregulated mRNA expression levels of ATPase sarcoplasmic/endoplasmic reticulum $\mathrm{Ca}^{2+}$ transporting 2, troponin-Il, troponin-Cl and troponin- $\mathrm{Tl}$ in young adult gastrocnemius muscle compared with the sucrose group. Nuclear factor- $\kappa B$-hypoxia inducible factor- $1 \alpha-T G F \beta$ receptor type-II-vascular endothelial growth factor staining indicated that ATG-125 decreased sucrose-induced chronic inflammation. ATG-125 was sufficient to prevent muscle atrophy, and this protective effect may be mediated through upregulation of AKT phosphorylation, upregulating the insulin growth factor-1R-insulin receptor substrate-PI3K-AKT pathway, which in turn resulted in a forkhead box O-dependent decrease in protein degradation pathways, including regulation of atrogin1 and E3 ubiquitin-protein ligase TRIM63. Peroxisome-proliferator activated receptor $\gamma$ coactivator $1 \alpha$ $(\mathrm{PGCl} \alpha)$ was decreased in young adult mice challenged with 
sucrose. ATG-125 treatment significantly increased PGC1 $\alpha$ and significantly increased UCP-1,2,3 expression levels, which suggested ATG-125 poised the mitochondria for uncoupling of respiration. This effect is consistent with the increased SIRT1 levels and may explain an increase in mitochondria biogenesis. Taken together, the present study showed that ATG-125, as an integrator of protein synthesis and degradative pathways, prevented muscle wasting.

\section{Introduction}

The largest protein reservoir in the body is the muscles. Muscles serve as a source of amino acids, which can be used for energy production by various organs during catabolic periods in human diseases (1). However, skeletal muscle can exhibit excessive protein degradation and the ensuing muscle loss can lead to muscle atrophy (2). Muscle atrophy, also known as muscle wasting disease, is a disease characterized by the loss of muscle tissue and progressive muscle weakening. If not sufficiently restored upon re-ambulation, a patient may experience muscle weakness, poorer physical function and eventually a reduced life span (1). Therefore, understanding the changes and mechanisms that occur during the early periods of muscle loss is of crucial importance in developing novel therapeutic targets.

Chronic inflammation, characterized by higher systemic cytokine and acute phase protein circulation $(3,4)$, is not only linked to aging (inflammaging) (5), but also loss of muscle mass (6). Inflammation impairs myogenesis and muscle homeostasis, activating the family of forkhead box O (FOXO) transcription factors, which then further promote skeletal muscle atrophy $(7,8)$. Conversely, it is well-established that inflammation serves an important role in developing insulin resistance (IR) (9-11), and this suggests that insulin signaling may modulate catabolic and anabolic pathways of muscle atrophy, potentially explaining the simultaneous occurrence of IR and muscle atrophy in several patients with type 2 diabetes $(12,13)$. Insulin-like growth factor 1 (IGF1) and/or insulin signaling can simultaneously inhibit protein breakdown and enhance muscle growth (14-16). Nevertheless, studies on AKT [also known as protein kinase B (PKB)] highlight another aspect of the IGF1 pathway in regulating muscle atrophy. AKT controls protein synthesis through the mechanistic target of rapamycin (mTOR) and regulates protein degradation through transcription factors in the FOXO family. However, pro-inflammatory cytokines (such as TNF- $\alpha$ ) contribute to inhibition of the IGF1-AKT pathway and IR (17-19). In addition, nuclear transcription factor nuclear factor $(\mathrm{NF})-\kappa \mathrm{B}$, which plays an important role as a mediator of immunity and inflammation, leads to an increase in the expression of inflammatory cytokines, particularly TNF- $\alpha$, during muscle wasting and cachexia (20).

Indeed, in human clinical trials, ingestion of sucrose alone by women results in an elevation of glucose concentration, followed by a high insulin response, reducing glucose concentrations to baseline and high glucose levels accompanied by a compensatory elevation in free fatty acid (FFA) concentration (21). In addition, FFAs serve a crucial role in the inflammatory process of cardiovascular diseases, as they serve as the building blocks for several components (cytokines) that are released through the portal vein and into the liver and then to other tissues $(21,22)$. Furthermore, increased circulating levels of FFAs is associated with a 7-fold increase in the risk of developing type 2 diabetes (22), due to impaired glucose-stimulated insulin secretion and resistance to the anti-lipolytic effect of insulin and increased adipose tissue mass (23). Therefore, effective strategies of improving IR and diabetes should include consideration of reduced intake of sucrose, reducing lipid metabolite accumulation of peripheral tissues and lowering the FFA levels. Increasing evidence has shown that sugars added to food products (including sucrose, glucose and fructose), increase the risk of development of inflammation, increased oxidative stress, dyslipidemia, hypertension and IR $(24,25)$. Indeed, high chronic sucrose intake accelerates sarcopenia through an alteration of postprandial stimulation of muscle protein synthesis in older male rats (26).

Herbal products contain several phytochemicals, such as carotenoids and polyphenols, alkaloids, flavonoids, glycosides, phenolic acids, saponins and lignans, all of which have proven health benefits (27-44). In Taiwan, herbal formula ATG-125 has been used in traditional therapy as it is believed to treat numerous ailments, including inflammation, muscle spasms, oral ulcers, wounds and rheumatic pain for several decades. However, there are no extensive studies assessing its effectiveness and its underlying mechanisms in muscle atrophy, to the best of our knowledge. ATG-125 is a the ethanolic extract of a herbal mixture consisting of Artemisia argyi (27), Morus alba L. (28), Leonurus japonicus Houtt. (29), Capsicum annuum L. (30,31), Lophatherum gracile Brongn. (32), Curcuma longa $(33,34)$ and Glycyrrhiza uralensis (35). Traditionally, these plants have been used for centuries for their antioxidant, anti-inflammatory and healing properties. Different classes of bioactive constituents are present in ATG-125, including several pseudoalkaloids, phenols and flavonoids, thus resulting in a pharmaceutical composition comprised of a mixture of chlorogenic acid (36-38), leonurine (29,39), schaftoside (40), rutin (36), isoschaftoside (41), isochlorogenic acid (38), quercetin $(42,43)$, apigenin $(43,44)$, glycyrrhizic acid $(35)$, curcumin $(33,34)$ and artemisetin $(27)$, all of which contribute to its medicinal properties.

In the present study, using transcriptomic screening/biomedical analysis for identification of potential muscle atrophy regulators in the phytochemical-rich formula ATG-125, the up- and downregulated genes in a high-sucrose intake model of muscle atrophy in mice were identified. The role of ATG-125 in the muscle response to sucrose was explored, with the present study being focused on muscle homeostasis and $\mathrm{Akt} / \mathrm{mitochondria}$ biogenesis signaling in young adult mice.

\section{Materials and methods}

Plant materials and extracts. ATG-125 is composed of seven herbal ingredients: i) Artemisia argyi leaves (154 g, Kuang Ying); ii) Morus alba L. leaves (154 g, Kuang Ying); iii) Leonurus japonicus Houtt. leaves (154 g, Kuang Ying); iv) Capsicum annuum L. leaves (77 g, Kuang Ying); v) Lophatherum gracile Brongn. leaves (77 g, Kuang Ying); vi) Curcuma longa root (31 g, San Hsing); and vii) Glycyrrhiza uralensis root (31 g, Jin Yi Hang). These 
herbs were purchased from local herbal medicine stores. To prepare the ethanolic extracts, $678 \mathrm{~g}$ of the herbal ingredients was soaked in 5 liters 95\% ethanol (Sigma-Aldrich; Merck KGaA) at room temperature for 1 day and filtered, then the filter procedure was repeated twice. Extraction was performed for $4 \mathrm{~h}$ at $70^{\circ} \mathrm{C}$ in a water bath and filtered, which was then repeated twice. All separate extractions were mixed and concentrated under reduced pressure. The yield was $99.66 \mathrm{~g}(14.7 \%)$ and ATG was stored at $4^{\circ} \mathrm{C}$ until required. Prior to use in the in vivo experiments, $1.5 \mathrm{~g}$ ATG-125 was dissolved in $100 \mathrm{ml}$ solvent (95\% olive oil and $5 \%$ glycerol).

High-performance liquid chromatography (HPLC). HPLC-UV analysis was performed using a Waters HPLC System (Waters Corporation), including a 600 quaternary pump, a Sugai U-620 column temperature controller, a 717 plus autosampler and a 996-photodiode array detector. The analytical conditions were set as follows: gradient elution by the mixture of mobile phases A $(0.085 \%$ phosphoric acid) and $\mathrm{B}$ (acetonitrile) at $0-10 \mathrm{~min}$ with a ratio of $85-90 \% \mathrm{~A}$ and $10-15 \% \mathrm{~B} ; 10-20$ min with a ratio of $80-85 \% \mathrm{~A}$ and $15-20 \% \mathrm{~B} ; 20-30$ min with a ratio of $60-80 \% \mathrm{~A}$ and $20-40 \% \mathrm{~B} ; 30-55$ min with a ratio of $35-60 \% \mathrm{~A}$ and $40-65 \%$ $\mathrm{B}$; 55-65 min with a ratio of $0-35 \% \mathrm{~A}$ and $65-100 \% \mathrm{~B}$; and $65-80$ min with the ratio of $0-90 \% \mathrm{~A}$ and $10-100 \% \mathrm{~B}$. The flow rate was kept constant at $1.0 \mathrm{ml} / \mathrm{min}$. The column temperature was controlled at $35^{\circ} \mathrm{C}$, the post time was $15 \mathrm{~min}$ and the injection volume was $20 \mu \mathrm{l}$. The UV wavelength for full detection was set at $200-600 \mathrm{~nm}$. The analytical column was a Cosmosil 5C18-MS-II column (particle size, $5 \mu \mathrm{m}$; column size, $4.6 \mathrm{~mm}$ internal diameter) x $250 \mathrm{~mm}$; Nacalai Tesque, Inc.) and a Lichrospher RP-18 end-capped column (particle size, $5 \mu \mathrm{m}$; column size, $4.0 \mathrm{~mm}$ internal diameter x10 mm; Merck KGaA) was used as a guard column.

Liquid chromatography-mass spectrometry (LC-MS) analysis and instrumental conditions. LC-MS was used to identify the major markers of bioactive substances. The system for analysis consisted of a LC-20AD UFLC system (Shimadzu Corporation) linked to a LCMS-2020 mass spectrometer. The running conditions were designed as follows: Gradient elution by the mixture of mobile phases A $(0.1 \%$ formic acid in water) and $\mathrm{B}$ (Acetonitrile) at minutes 0-65, with the ratio of $90-0 \%$ in $\mathrm{A}$ and $10-100 \%$ in $\mathrm{B}$; at minutes $65-80$ with the ratio of $0-90 \%$ in A and $100-10 \%$ in B. The flow rate was fixed at $1 \mathrm{ml} / \mathrm{min}$ and the column temperature was maintained at $35^{\circ} \mathrm{C}$. The injection volume was adjusted to $20 \mu \mathrm{l}$ and the analytical column used was a Cosmosil 5C18-MS-II (5 $\mu \mathrm{m}, 4.6 \times 250 \mathrm{~mm}$; Nacalai Tesque, Inc.). Dual ion modes [electrospray ionization, $(\mathrm{ESI})(+)$ and $(-)$ ] were used in MS detection and the transmission of $(\mathrm{M}+\mathrm{H})+$ and (M-H)-was set as the optimum condition. The MS detection was set to full scan range $(50-1,200 \mathrm{~m} / \mathrm{z})$. The interface voltages were set at $4.5 \mathrm{kV}$ for $\mathrm{ESI}(+)$ and $-4.5 \mathrm{kV}$ for ESI(-). With nitrogen as the nebulizing and drying gas, the flow rate was set at 1.5 and $15 \mathrm{l} / \mathrm{min}$, respectively. Nitrogen gas temperature was set at $350^{\circ} \mathrm{C}$. Desolvation line temperature was set at $300^{\circ} \mathrm{C}$ and heat block temperature was maintained at $500^{\circ} \mathrm{C}$. Chromatographic fingerprint analysis was performed using
3D HPLC, which was primarily performed as described previously $(45,46)$.

Animals. A total of 15 male young adult C57BL/6J mice (age, 6 months; weight, 24-26 g) were obtained from the National Laboratory Animal Center (Taiwan) and provided ad libitum access to standard chow and water and housed (40-60\% humidity) with a controlled temperature $\left(26^{\circ} \mathrm{C}\right)$ and a 12 -h light/dark cycle (lights off at $7 \mathrm{pm}$ ). Animal studies were performed according to previous studies $(47,48)$ and were approved by the Chang Gung University Animal Care and Use Committee (approval no. CGU108-111; Taoyuan, Taiwan). Mice were randomized into three groups ( $\mathrm{n}=5$ per group): i) Young adult mice; ii) young adult mice fed with $30 \%$ sucrose for 28 days; and iii) young adult mice fed with $30 \%$ sucrose and ATG-125 (0.2 ml applied each day to the abdominal skin) on days 15-28. All mice were maintained on their assigned diet until they were sacrificed by $\mathrm{CO}_{2}\left(\mathrm{CO}_{2}\right.$ flow rate, volume displacement at $70 \%$ of the cage $\mathrm{vol} / \mathrm{min}$ ).

Western blot analysis. Gastrocnemius muscles were harvested immediately, flash frozen in liquid nitrogen and stored at $-80^{\circ} \mathrm{C}$. Protein was extracted from muscle tissues using distilled water using a T-PER ${ }^{\mathrm{TM}}$ Tissue Protein Extraction Reagent $(0.30 \mathrm{mg}$ tissue $/ 200 \mu \mathrm{l}$; cat. no. 78510 ; Thermo Fisher Scientific, Inc.) containing protease inhibitors $(1 \mu \mathrm{l} / \mathrm{ml}$; cat. no. SI-P8340; Sigma-Aldrich; Merck KGaA), and the protein concentration was determined using a Bio-Rad protein assay (Bio-Rad Laboratories, Inc.). Blocking was performed using $5 \% \mathrm{w} / \mathrm{v}$ nonfat dry milk, $1 \mathrm{X}$ TBS with $0.1 \%$ Tween-20 at room temperature for $1 \mathrm{~h}$. Protein $(50 \mu \mathrm{g} /$ lane $)$ was run on a $10 \%$ SDS-PAGE gel and subsequently transferred to a PVDF membrane. For immunoblotting, membranes were incubated with diluted primary antibody in $5 \% \mathrm{w} / \mathrm{v}$ nonfat dry milk, $1 \mathrm{X}$ TBS with $0.1 \%$ Tween- 20 at $4^{\circ} \mathrm{C}$ with gentle shaking overnight. The following primary antibodies were used: Phosphorylated (p)-AMP-activated protein kinase (1:500; p-AMPK; Thr 172; cat. no. sc-33524; Santa Cruz Biotechnology, Inc.), AMPK (1:500; cat. no. sc-74461; Santa Cruz Biotechnology, Inc.), p-FOXO3a (1:500; S253; cat. no. ab47285; Abcam), FOXO3a (1:500; cat. no. ab17026; Abcam), muscle RING-finger protein-1 (MuRF1; 1:1,000; cat. no. ab77577; Abcam), $\beta$-actin (1:1,000; cat. no. MAB1501; MilliporeSigma), peroxisome proliferator-activated receptor (PPAR) $\gamma$ coactivator $1-\alpha$ (PGC1 $\alpha ; 1: 1,000$; cat. no. ab54481; Abcam), Sirtuin 1 (SIRT1; 1:1,000; cat. no. ab110304; Abcam), uncoupling protein (UCP)1 (1:1,000; cat. no. ab10983; Abcam), UCP2 (1:500; cat. no. ab203244; Abcam) and UCP3 (1:500; cat. no.ab10985; Abcam). The primary incubation was followed by incubation with secondary antibodies in $5 \% \mathrm{w} / \mathrm{v}$ nonfat dry milk, $1 \mathrm{X}$ TBS with $0.1 \%$ Tween-20 at room temperature with gentle shaking for $1 \mathrm{~h}$. The following secondary antibodies were used: Goat anti-mouse IgG antibody, HRP-conjugated (1:1,000; cat. no. Ap124P; MilliporeSigma), goat anti-rabbit IgG antibody, biotin-SP (long spacer)-conjugated (1:1,000; cat. no. 111-065-003; Jackson ImmunoResearch Laboratories, Inc.) and goat anti-rat IgG antibody, biotin-SP-conjugated (1:1,000; cat. no. 112-065-003; Jackson ImmunoResearch Laboratories, Inc.). Signals were visualized using a chemiluminescence system (Amersham; Cytiva) according to the 
manufacturer's instructions. The optical densities of the bands were semi-quantified using ImageJ version 1.53 (National Institutes of Health).

Histology, immunohistochemistry and immunofluorescence. Muscle tissue was fixed in $4 \%$ paraformaldehyde solution for 3 days, processed for paraffin embedding and cut into $5-\mu \mathrm{m}$ thick sections at room temperature. For histology, sections were stained with hematoxylin $(2 \mathrm{~g} / \mathrm{l})$ for $15 \mathrm{~min}$ and with eosin $(0.1 \%$ in $0.0003 \%$ acetic acid) for $10 \mathrm{~min}$ at room temperature (49). Before immunostaining, slides were heated in an oven at $65^{\circ} \mathrm{C}$ for $15 \mathrm{~min}$ and the sections were deparaffinized using xylene and placed in a series of graded alcohol solutions, as routine dehydrated processing. The endogenous peroxidase activity was blocked by hydrogen peroxidase treatment $(3 \%)$. The sections were then washed with distilled water and transferred to TBST (0.5\% Tween-20; pH 7.4) containing 5\% normal goat serum (cat. no. G9023; Sigma-Aldrich; Merck KGaA) for 30-50 min at room temperature. Next, sections were incubated at room temperature for $2 \mathrm{~h}$ with antibodies against p-AMPK (1:100), glucose transporter type 4 insulin-responsive (Glut4; 1:80; cat. no. ab33780; Abcam), p-FOXO3a (1:100), FOXO3A (1:100), MuRF1(1:100), NF-кB (1:100; cat. no. sc-8008; Santa Cruz Biotechnology, Inc.), hypoxia inducible factor-1 $\alpha$ (HIF-1 $\alpha$; 1:100; cat. no. ab179483; Abcam), vascular endothelial growth factor (VEGF; 1:100; cat. no. ab69479; Abcam), TGF $\beta$ receptor type-II (TGF $\beta$ RII; 1:100; cat. no. sc-17791; Santa Cruz Biotechnology, Inc.), p-p53 (S15; 1:100; cat. no. ab1431; Abcam), Ki67 (1:100; cat. no. AB9260; MilliporeSigma), PGC1 $\alpha$ (1:100), UCP1 (1:100), UCP2 (1:100; cat. no. ab97931; Abcam) UCP3 (1:100), p-insulin-like growth factor 1 receptor (IGF1R; Y1161; 1:100; cat. no. ab39398; Abcam), p-insulin receptor substrate 1 (IRS1; Tyr 989; 1:100; cat. no. sc-17200; Santa Cruz Biotechnology, Inc.), p-PI3K (p85; 1:100; cat. no. GTX111068; GeneTex, Inc.), p-AKT (Ser473; 1:100; cat. no. sc-7985-R; Santa Cruz Biotechnology, Inc.), p-mTOR (Ser 2448; 1:100; cat. no. ab109268; Abcam), p-ribosomal S6 kinase (S6K; Thr389; 1:100; cat. no. 9234; Cell Signaling Technology, Inc.), p-eukaryotic translation initiation factor 4E-binding protein 1 (4EBP1; Thr37/461; 1:100; cat. no. 2855; Cell Signaling Technology, Inc.) and MitoTracker $^{\mathrm{TM}}$ Red CMXRos (1:100; cat. no. M7512; Thermo Fisher Scientific, Inc.). Sections were incubated with the appropriate secondary antibodies, including Alexa Fluor 488-conjugated rabbit IgG (heavy chain + light chain) highly cross adsorbed (1:100; cat. no. A11034; Thermo Fisher Scientific, Inc.), Alexa Fluor Plus 647-conjugated donkey anti-rabbit (1:100; cat. no. A32795; Thermo Fisher Scientific, Inc.), goat-anti-mouse IgG HRP-conjugated (H+L; 1:100; cat. no. G-21040; Thermo Fisher Scientific, Inc.) or goat anti-rabbit IgG HRP-conjugated (H+L; 1:100; cat.no. G-21234; Thermo Fisher Scientific, Inc.) antibodies for $1 \mathrm{~h}$ at room temperature. All antibodies were diluted in $2 \%$ non-immune goat serum (cat. no. G9023; Sigma-Aldrich; Merck KGaA) in PBS with Tween-20 (0.05\%) buffer at room temperature. Sections were incubated with DAB (MilliporeSigma) for 5-10 min, and hematoxylin (Sigma-Aldrich; Merck KGaA) for $3 \mathrm{~min}$, or DAPI (1:10,000; cat. no. 62248; Thermo Fisher Scientific, Inc.) was used for nuclear staining, and both staining incubations were performed at room temperature. The optical observed of the stain using CellSens Dimension software package (Olympus Corporation) and an inverted fluorescence microscope (Olympus IX71; Olympus Corporation).

$R N A$ isolation and reverse transcription-quantitative (RT-q) $P C R$. Total RNA was isolated from $0.2 \mathrm{~g}$ muscle tissues using TRIzol $^{\circledR}$ (Thermo Fisher Scientific, Inc.) and an RNeasy kit (Thermo Fisher Scientific, Inc.) according to the manufacturer's protocol. For qPCR, equal quantities of RNA were reverse transcribed to cDNA using a High-Capacity cDNA Reverse Transcription kit (Thermo Fisher Scientific, Inc.) according to the manufacturer's instructions. The following thermocycling conditions were used for qPCR: Initial denaturation at $95^{\circ} \mathrm{C}$ for $5 \mathrm{~min} ; 35$ of cycles of denaturation at $94^{\circ} \mathrm{C}$ for $30 \mathrm{sec}$, annealing at $55.8^{\circ} \mathrm{C}$ for $30 \mathrm{sec} 0.5 \mathrm{~min}$, elongation at $72^{\circ} \mathrm{C}$ for $0.5 \mathrm{~min}$; final extension at $72^{\circ} \mathrm{C}$ for $30 \mathrm{sec} 0.5 \mathrm{~min}$ ); and holding at $4^{\circ} \mathrm{C}$. The expression levels of Atrogin-1, NADPH oxidase (Nox)-1, Sterol regulatory element-binding protein (Srebp)-1, Carnitine palmitoyltransferase I (Cpt-1), Ppar $\alpha$, Ppar $\gamma$, acyl-CoA oxidase (Aco), mitochondrial transcription factor A (Tfam), Sirtl, Pgcl $\alpha$, Nuclear respiratory factor 1 (Nrfl), Igflr, Irs1, Pi3k, Akt, mTOR, S6k, 4ebpl and Gapdh were evaluated using SYBR Green PCR MasterMix (Roche Diagnostics GmbH). Gapdh was used as the loading control. Relative expression was calculated using the $2^{-\Delta \Delta \mathrm{Cq}}$ method with normalization to constitutive genes (50). The sequences of the primers used for RT-qPCR are listed in Table SI (51-77).

RNA isolation and library preparation for RNA-Seq. Total RNA was extracted from gastrocnemius muscles using a Qiagen RNeasy Mini Kit (cat. no. 74004; Qiagen GmbH). RNA purity and quality were evaluated using a SimpliNano ${ }^{\mathrm{TM}}$ Biochrom Spectrophotometer (cat. no. 29061712; Biochrom, Ltd.). A total of $1 \mu \mathrm{g}$ total RNA/sample was used as input material for the RNA sample preparations. In order to select cDNA fragments of $\sim 150 \mathrm{bp}$ in length, the library fragments were purified using the KAPA Pure Beads System (cat. no. 07983298001; KAPA Biosystems; Roche Diagnostics $\mathrm{GmbH}$ ). cDNA was synthesized using a KAPA mRNA HyperPrep Kit (cat.no. 08098123702; Kapa Biosystems; Roche Diagnostics $\mathrm{GmbH}$ ) according to the manufacturer's protocol and index codes. The clustering of the index-coded samples was performed on a cBot Cluster Generation System using PE Cluster Kit cBot-HS (cat. no. PE-401-3001; Illumina, Inc.) according to the manufacturer's instructions. Following cluster generation, the library preparations were sequenced using an Illumina platform (NovaSeq 6000 S4 Reagent Kit v1.5; 300 cycles; cat. no. 20028312; Illumina, Inc.) and 125 bp/150 bp paired-end reads were generated. The original data obtained by high-throughput sequencing (Illumina NovaSeq 6000 platform; Illumina, Inc.) were transformed into raw sequenced reads by CASAVA base calling and stored in the FASTQ format, and FastQC, MultiQC and ReSeqtools were used to check FASTQ files for quality (78). The final library qubit concentration was $3.74 \mathrm{ng} / \mu \mathrm{l}$.

Raw paired-end reads were filtered by Trimmomatic (version 0.38) (79). Next, the genome alignment methods from hierarchical indexing for spliced alignment of transcripts (HISAT2; version 2.1.0) (80), RSeQC (version 4.0.0) (81), 
Qualimap (version 2.2.1) (82) and featureCounts (version 2.0.0) (83) were used. Gene expression values were represented using the normalization techniques provided by each algorithm: Fragments per Kilobase of Mapped reads (FPKM), Transcripts per Million (TPM), Trimmed Mean of $M$ values (TMM from edgeR) and Relative Log Expression (RLE from DESeq2) $(78,84)$. Gene function of the mapped reads (unique transcripts) was annotated based on the NCBI non-redundant protein sequences database (85). Resulting P-values were adjusted using the Benjamini and Hochberg's approach for controlling the false discovery rate (FDR) (86). Differentially expressed genes (DEGs) with a threshold FDR adjusted, P.adjust $<0.05$ and fold-change $2(\log 2> \pm 1)$ were selected for further analysis. Principal component analysis (PCA) (84), volcano plots ggplot2 version 3.3.3 (R package) (87), Gene Ontology (GO) $(88,89)$, Kyoto Encyclopedia of Genes and Genomes (KEGG) (89), Disease Ontology (DO) (90) and Gene Set Enrichment Analysis (GSEA) (91) enrichment of the DEGs were analyzed. ClusterProfiler package (version 3.14.3) supported the enrichment analysis of GO and KEGG with either hypergeometric tests or GSEA (91). Furthermore, the function emapplot and heatmap diagram were also from the enrichplot package of ClusterProfiler (91).

Statistical analysis. All data are presented as the mean \pm standard error of the mean. Statistical analysis was performed using SPSS version 21.0 (IBM Corp.). All experiments were repeated three times for data analysis. All data were analyzed using a one-way ANOVA followed by a Bonferroni's post hoc test. $\mathrm{P}<0.05$ was considered to indicate a statistically significant difference.

\section{Results}

LC-MS analysis of the composition of ATG-125. A typical LC-MS chromatographic fingerprint profile of ATG-125 was obtained (Fig. 1) and 10 major components were identified. The well-separated peaks with retention times of $60 \mathrm{~min}$ were identified as follows: i) Chlorogenic acid (12.82 min); ii) Leonurine (20.375 min); iii) Rutin (27.060 min); iv) Isoschaftoside (31.078 min); v) Isochlorogenic acid (3,5-Dicaffeoylquinic acid, $31.52 \mathrm{~min})$; vi) Quercetin (36.836 min); vii) Apigenin (39.271 $\mathrm{min})$; viii) Glycyrrhizic acid (41.667 min); ix) Curcumin (50.946 min); and $x$ ) Artemisetin (52.064) (Table I). The structure of compounds (Fig. 1A-D) and the 3D-HPLC fingerprint (Fig. 1E and F) are shown.

Global transcriptome characteristics of ATG-125 on sucroseinduced young adult muscle atrophy in mice. To investigate the molecular-level changes in the gastrocnemius muscles of sucrose-induced muscle atrophy in mice treated with or without ATG-125, RNA-seq analysis of the gastrocnemius muscle lysates was performed (Fig. 2A). Principal Component Analysis revealed distinct gene expression profiles for the three different groups (Fig. 2B). The gene count data were used to analyze differences in gene expression using DESeq2. A total of 136 DEGs were identified, including 18 significantly upregulated and 118 significantly downregulated genes between the ATG-125 treated and untreated sucrose-induced groups. A volcano plot of the DEGs is shown in Fig. 2C. The top 18 upregulated DEGs were identified: Acyl-CoA thioesterase 2, angiopoietin-like 4, adipocyte-related X-chromosome expressed sequence 2, expressed sequence BB365896, bone morphogenetic protein $3, \mathrm{CDC} 14$ cell division cycle $14 \mathrm{~A}$, ChaC cation transport regulator 1 , cell death-inducing DFFA-like effector c, RIKEN cDNA E230001N04 gene, growth arrest and DNA-damage-inducible $45 \mathrm{~b}, \mathrm{G}$ protein-coupled receptor 160, methyltransferase like 21E, Nmrk2, pyruvate dehydrogenase kinase isoenzyme 4, pyruvate dehydrogenase phosphatase catalytic subunit 2 , solute carrier family 7 (cationic amino acid transporter, $\mathrm{y}+$ system) member $10, U c p 3$ and zinc finger and BTB domain-containing protein 16, all of which were significantly increased in the ATG-125 treated sucrose-induced mice compared with the sucrose challenged mice (Table II). The top 14 downregulated DEGs were $\alpha$-2-HS-glycoprotein, apolipoprotein (Apo)a1, Apoa2, Apoc3, cytochrome P450 family 3 subfamily A polypeptide 11, fatty acid binding protein 1, fibrinogen $\beta$ chain, methionine adenosyltransferase 1a, major urinary protein 3 , serine (or cysteine) peptidase inhibitor clade A member (Serpina)1b, Serpinald, Serpinale, Serpina $3 k$ and solute carrier organic anion transporter family member 1B2, all of which were significantly decreased in the ATG-125 treated sucrose induced mice compared with the sucrose-challenged mice (Table III).

Given that the distinction between the ATG-125 treated and untreated clusters were most evident in the gastrocnemius muscle, the muscle contraction genes in gastrocnemius muscle that varied according to ATG-125 treatment were probed. To further determine the differences between the two biological states, GSEA was performed using the ClusterProfiler package. A heatmap of the muscle contraction genes showed that they displayed a steady decreasing trend with ATG-125 treatment. Of these, ATG-125 treatment significantly downregulated the mRNA levels of ATPase sarcoplasmic/endoplasmic reticulum $\mathrm{Ca}^{2+}$ transporting 2, troponin I slow skeletal muscle (Tnnil), troponin T cardiac muscle (Tnnt2) and troponin T slow skeletal muscle (Tnntl) in gastrocnemius muscle compared with the sucrose group (Fig. 2D).

Effect of ATG-125 on DEGs and functional variation in sucrose-induced young adult muscle atrophy in mice. To further analyze and classify the biological function of DEGs, GO functional analysis was performed using the ClusterProfiler package. GO analysis of the DEGs were sub-divided into three categories: Biological process (BP; Fig. 3A), molecular function (MF; Fig. 3B) and cellular component (CC; Fig. 3C) and only significant GO terms with $\mathrm{P}$-adjust $<1$ were considered. Top 10 (including downregulated and upregulated) gene-enriched GO terms were identified and are shown in Fig. 3. In the BP group, the top $2 \mathrm{GO}$ terms were 'negative regulation of hydrolase activity' and 'negative regulation of proteolysis' (Fig. 3A). In the MF group the top GO term was 'enzyme inhibitor activity' (Fig. 3B). In the CC group, the top 2 GO terms were 'collagen-containing extracellular matrix' and 'extracellular matrix' (Fig. 3C).

To analyze the functional significance of the proteins with altered expression corresponding to the ATG-125 treatment, GO Enrichment Map (emapplot) was used to identify functional networks, which were visualized using ClusterProfiler. Fig. 3D provides a graphical overview of the GO Enrichment 
A

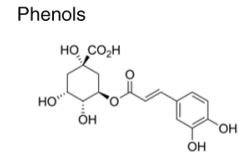

(1) Chlorogenic acid

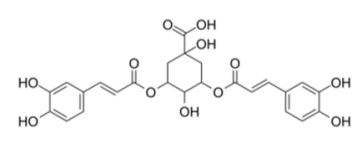

(5) 3,5-Dicaffeoylquinic

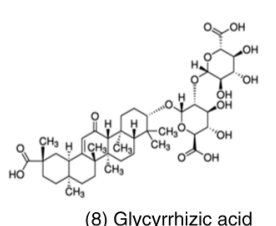

B<smiles>COc1cc(C(=O)OCCCCN=C(N)N)cc(OC)c1O</smiles>

(2) Leonurine
C Flavonoid<smiles>COc1cc(/C=C/C(=O)CC(=O)/C=C/c2ccc(O)c(OC)c2)ccc1O</smiles>

(9) Curcumin<smiles>CC(C)CCCCCCO</smiles>

(3) Rutin

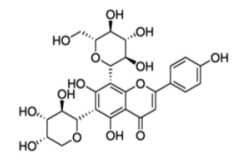

(4) Isoschaftoside

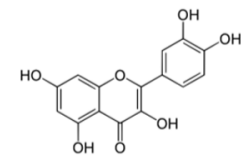

(6) Quercetin

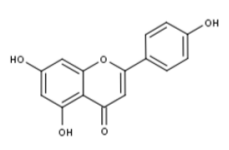

(7) Apigenin

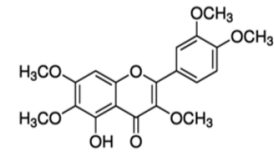

(10) Artemisetin

E

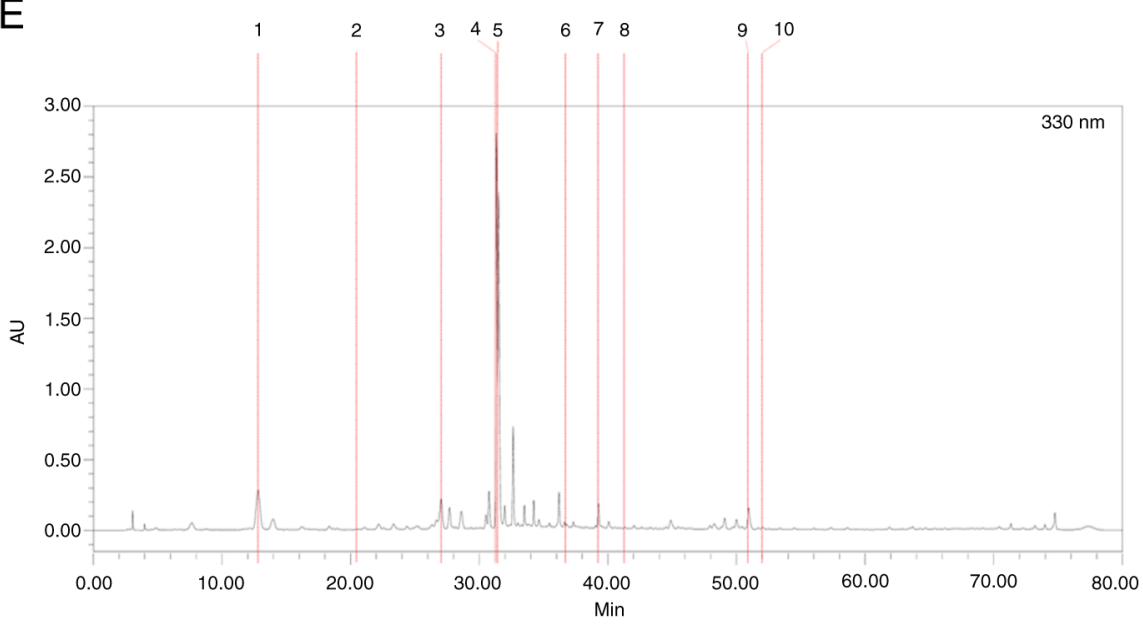

$\mathrm{F}$

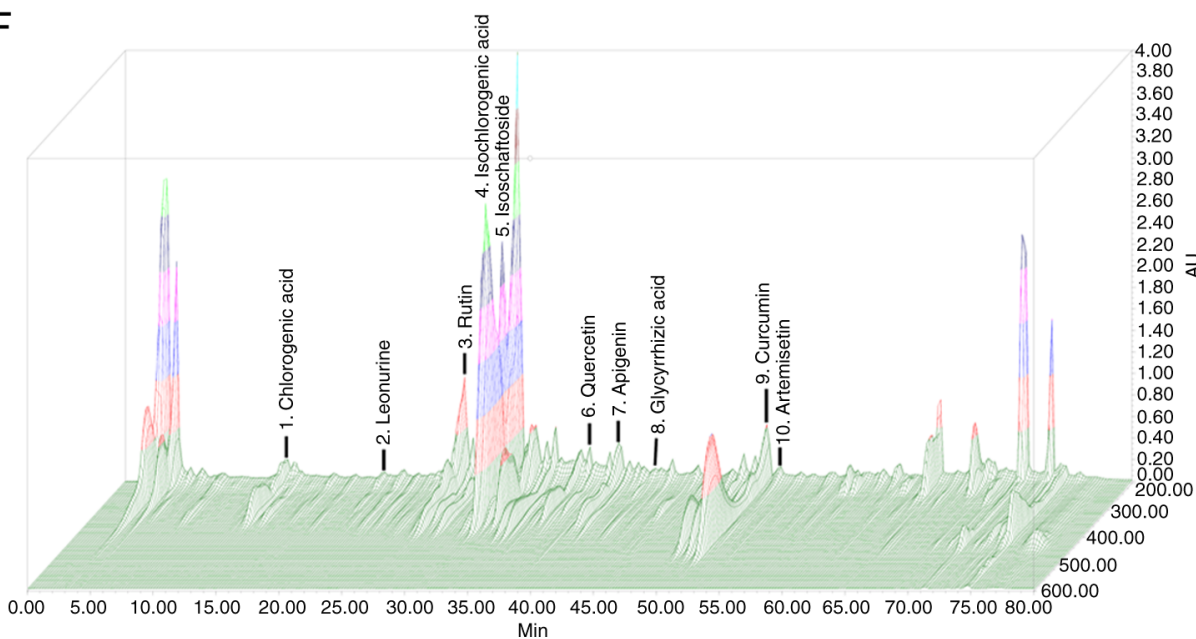

Figure 1. Chromatogram of the herbal formula ATG-125 obtained by LC-MS. (A-D) Structures of the compounds identified by LC-MS. Chlorogenic acid, Leonurine, Rutin, Isoschaftoside, Isochlorogenic acid (3,5-Dicaffeoylquinic acid), Quercetin, Apigenin, Glycyrrhizic acid, Curcumin and Artemisetin. (E) HPLC and (F) 3D-HPLC fingerprints. Chromatographic fingerprint analysis was performed using HPLC and LC-MS. HPLC, high performance liquid chromatography; LC-MS, liquid chromatography-mass spectrometry.

Map results and indicates that the altered behavior in response to ATG-125 treatment was observed for different groups of proteins. Using the GO Enrichment Map plugin gene function prediction, the gene interaction network was divided into seven subgroups, which were associated with: i) ' $\alpha$-amino acid metabolic process'; ii) 'cellular amino acid metabolic process'; iii) 'fibrinolysis'; iv) 'negative regulation of hydrolase activity'; v) 'negative regulation of peptidase activity'; vi) 'negative regulation of proteolysis'; and vii) 'small molecule catabolic process'. This category emapplot was supported by the GO analysis (Fig. 3D).

GO enrichment and DO analysis of ATG-125-regulated DEGs. For GO analysis, 2,203 DEGs from gastrocnemius muscle were classified into three $\mathrm{GO}$ categories (BP, CC and MF). The top 30 (downregulated and upregulated) gene-enriched GO terms were identified and are shown in Fig. 4A. GO enrichment analysis was performed to 
Table I. Percentage of chlorogenic acid, leonurine, rutin, isoschaftoside, isochlorogenic acid (3,5-dicaffeoylquinic acid), quercetin, apigenin, glycyrrhizic acid, curcumin and artemisetin.

\begin{tabular}{|c|c|c|c|c|c|}
\hline No. & $\begin{array}{l}\text { Retention } \\
\text { time (min) }\end{array}$ & Compound & $\begin{array}{l}\text { Wavelength } \\
\text { (nm) }\end{array}$ & $\begin{array}{l}\text { Weight of compound } \\
\text { in ATG-125 }(\mathrm{mg} / \mathrm{g})\end{array}$ & $\begin{array}{l}\text { Percentage of compound } \\
\text { in ATG-125 (\%) }\end{array}$ \\
\hline 1 & 12.82 & Chlorogenic acid & 327 & 3.82 & 0.38 \\
\hline 2 & 20.375 & Leonurine & 280 & 0.40 & 0.04 \\
\hline 3 & 27.060 & Rutin & 350 & 3.64 & 0.36 \\
\hline 4 & 31.078 & Isoschaftoside & 327 & 20.34 & 2.03 \\
\hline 5 & 31.52 & $\begin{array}{l}\text { Isochlorogenic acid } \\
\text { (3,5-Dicaffeoylquinic acid) }\end{array}$ & 327 & 11.83 & 1.18 \\
\hline 6 & 36.836 & Quercetin & 370 & 0.06 & 0.01 \\
\hline 7 & 39.271 & Apigenin & 330 & 0.64 & 0.06 \\
\hline 8 & 41.667 & Glycyrrhizic acid & 250 & 0.89 & 0.09 \\
\hline 9 & 50.946 & Curcumin & 430 & 2.98 & 0.30 \\
\hline 10 & 52.064 & Artemisetin & 350 & 0.11 & 0.01 \\
\hline
\end{tabular}

determine whether the genes in the GO terms were enriched in 'negative regulation of hydrolase activity', 'negative regulation of peptidase activity', 'enzyme inhibitor activity', 'endopeptidase inhibitor activity', 'negative regulation of proteolysis', 'endopeptidase regulator activity', 'peptidase inhibitor activity', 'peptidase regulator activity' and 'serine-type endopeptidase inhibitor activity'.

Next, a single asserted classification was used to include multiple-inferred mechanistic disease classifications from DO analysis of DEGs, which was performed using the top 10 DO terms in Fig. 4B. The results showed that Serpina 3 and its interacting genes were significantly enriched in deep vein thrombosis, blood coagulation disorders, vascular diseases, ischemic stroke and age-related macular degeneration based on DO analysis (Fig. 4B).

KEGG analysis of DEGs following ATG-125 treatment of sucrose-induced muscle atrophy. The KEGG enrichment analysis classified the top 10 downregulated and upregulated pathways of the DEGs (Fig. 5A and B). The top downregulated 4 enriched pathways in the gastrocnemius muscle were 'complement and coagulation cascades', 'cholesterol metabolism', 'biosynthesis of amino acids' and 'PPAR signaling pathway'. The top upregulated 4 enriched pathways in the gastrocnemius muscle were ' cell cycle', ' transcriptional misregulation in cancer', ' fatty acid elongation' and 'protein export' (Fig. 5A and B). Examination of the KEGG enrichment analysis showed that $85-90 \%$ of the expressed genes were significantly downregulated in the ATG-125 treated sucrose-induced mice (Fig. 5C). These enriched terms highlighted the mechanisms regulated by ATG-125 and provide direction for further research.

ATG-125 attenuates the AMPK/FOXO3a/MuRF1 signaling pathway in sucrose-induced gastrocnemius muscle atrophy. In the present study, the body weight of mice fed sucrose did not differ significantly compared with the young adult group (Fig. 6A). Mice fed sucrose exhibited a significant decrease in gastrocnemius muscle weight, as well as in the ratio of gastrocnemius muscle to body weight compared with the young adult group (Fig. 6B and C), whereas ATG-125 treatment significantly increased muscle weight and the ratio of body weight to muscle compared with the sucrose group (Fig. 6B and C). Mice fed sucrose exhibited increased quantities of pre-necrotic hyaline and necrotic fibers, and ATG- 125 treatment notably reduced sucrose-induced muscle atrophy (Fig. 6D). Sucrose markedly increased gastrocnemius muscle GLUT- 4 content but this markedly decreased after ATG-125 treatment (Fig. 6D).

To further confirm whether sucrose-induced gastrocnemius muscle weight loss was associated with protein degradation, the activity of the AMPK/FOXO3a/MuRF1 signaling pathway in gastrocnemius muscle was assessed. Immunohistochemical and western blot analysis showed p-FOXO3a/OXO3a expression was significantly reduced in the sucrose fed group compared with the control, and increased in the ATG-125 treatment group. Furthermore, high p-AMPK and MuRF1 protein levels, and high Atrogin-1 and Klotho mRNA expression levels in the gastrocnemius muscle of young adult mice fed sucrose compared with the young adult group (Fig. 6D-G). ATG-125 significantly reduced AMPK/FOXO3a/MuRF1 signaling pathway activity compared with the sucrose group, suggesting that ATG-125 improved protein degradation through inhibiting AMPK/FOXO3a/MuRF1 signaling in sucrose-induced muscle atrophy.

ATG-125 improves chronic inflammation in sucrose-induced gastrocnemius muscle atrophy. To determine the dynamics of recovery from sucrose-induced gastrocnemius muscle atrophy, the mechanisms of chronic inflammation in sucrose-fed young adult mice treated with ATG-125 were determined. Immunohistochemical analysis showed HIF-1 $\alpha$, VEGF, TGF $\beta$ RII, NF- $\kappa$ B, p-p53 and Ki67 protein levels, as well as Nox2, Srebp-1, Cpt-1, Ppara, Ppar- $\gamma$, Aco, Tnfa, Il-6, Il-1 $\beta$ and $C r p$ mRNA expression levels were higher in the sucrose-induced mice (Fig. 7A-E). ATG-125 significantly decreased HIF- $1 \alpha$ and NF- $\kappa$ B mediated inflammation signaling compared with sucrose-challenged mice. Thus, ATG-125 is an important regulator of chronic inflammation. 
A

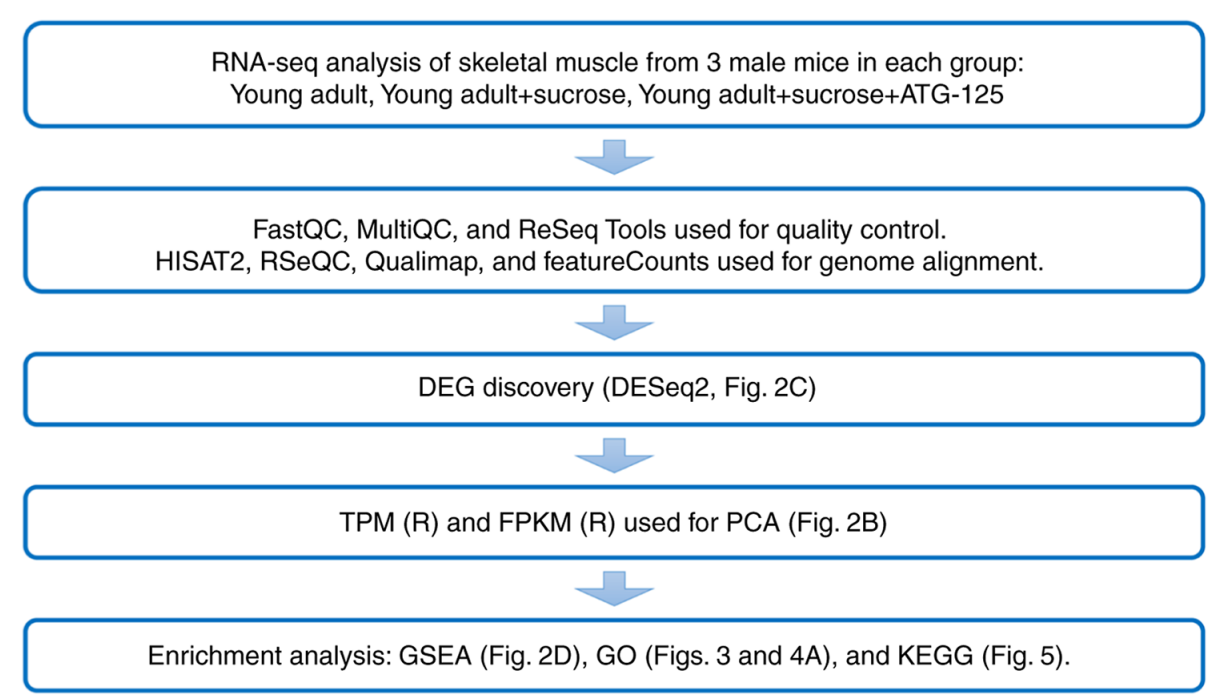

$B_{3 D}$ PCA

Young adult+sucrose+ ATG-125 vs. Young adult+sucrose

C DEG_DESeq2_volcano_plot

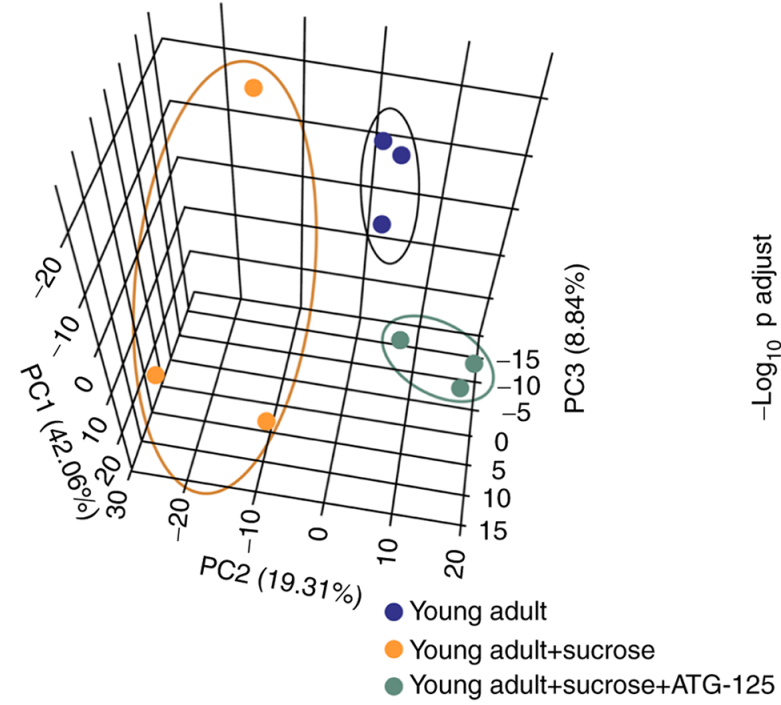

Young adult+sucrose+ATG-125 vs. Young adult+sucrose

D Regulation of muscle contraction heatmap

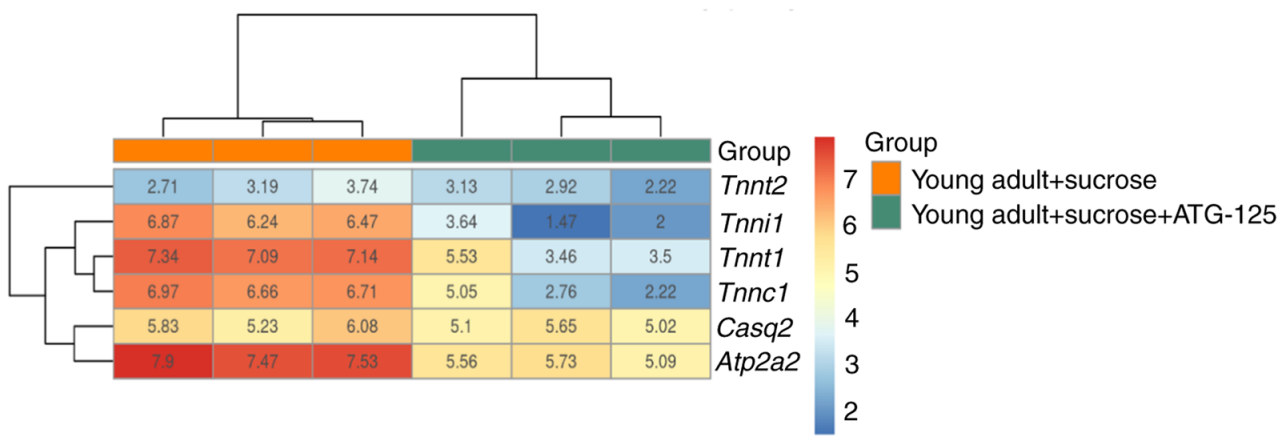

Figure 2. Transcriptome characteristics of mice muscles in the sucrose-induced muscle atrophy group with or without ATG-125 treatment. (A) RNA-seq analysis workflow. (B) PCA showing overall gene expression patterns in young adult mice, sucrose-induced muscle atrophy mice and ATG-125 treated sucrose-induced muscle atrophy mice. (C) Volcano plot of the DEGs. (D) Heatmap showing genes associated with muscle contraction. PCA, Principle Component Analysis; DEG, differentially expressed gene; FPKM, Fragments per Kilobase of Mapped reads; TPM, Transcripts per Million; GO, Gene Ontology; KEGG, Kyoto Encyclopedia of Genes and Genomes; GSEA, Gene Set Enrichment Analysis; Tnnt2, troponin T cardiac muscle; Tnnt1, troponin T slow skeletal muscle; Tnnil, troponin I slow skeletal muscle; Casq2, calsequestrin 2; Atp2a2, ATPase sarcoplasmic/endoplasmic reticulum Ca2+ transporting 2.

ATG-125 improves sucrose-induced mitochondrial dysfunction during muscle atrophy in young adult mice. Next, the mechanistic underpinnings of the sucrose-induced mitochondrial dysfunction observed in muscle tissue of sucrose-fed mice under ATG-125 treatment were investigated. Mice fed sucrose exhibited lower SIRT1, PGC1 $\alpha$, UCP1, UCP2 
Table II. Top 18 upregulated differentially expressed genes.

\begin{tabular}{|c|c|c|c|c|}
\hline Symbol & Description & $\log 2$ Fold-change & P-value & padj \\
\hline Acot 2 & Acyl-CoA thioesterase 2 & 1.34541 & $3.05 \times 10^{-8}$ & $6.31 \times 10^{-5}$ \\
\hline Angptl4 & angiopoietin-like 4 & 2.70437 & $2.45 \times 10^{-5}$ & 0.00680 \\
\hline Arxes2 & Adipocyte-related X-chromosome expressed sequence 2 & 3.62662 & 0.00014 & 0.02329 \\
\hline BB365896 & Expressed sequence BB365896 & 1.61957 & 0.00020 & 0.02922 \\
\hline Bmp3 & Bone morphogenetic protein 3 & 2.69999 & 0.00037 & 0.04651 \\
\hline$C d c 14 a$ & CDC14 cell division cycle $14 \mathrm{~A}$ & 1.10848 & 0.00011 & 0.02031 \\
\hline Chacl & ChaC cation transport regulator 1 & 1.87761 & $4.18 \times 10^{-5}$ & 0.00997 \\
\hline Cidec & Cell death-inducing DFFA-like effector c & 3.62151 & 0.00029 & 0.03906 \\
\hline E230001N04Rik & RIKEN cDNA E230001N04 gene & 2.43998 & $3.86 \times 10^{-6}$ & 0.00175 \\
\hline Gadd45b & Growth arrest and DNA-damage-inducible $45 \mathrm{~b}$ & 1.03662 & 0.00039 & 0.04830 \\
\hline Gpr160 & G protein-coupled receptor 160 & 1.79903 & 0.00019 & 0.02845 \\
\hline Mettl21e & Methyltransferase like 21E & 1.37094 & 0.00023 & 0.03285 \\
\hline Nmrk2 & Nicotinamide riboside kinase 2 & 1.15367 & $3.47 \times 10^{-6}$ & 0.00162 \\
\hline$P d k 4$ & Pyruvate dehydrogenase kinase isoenzyme 4 & 2.53921 & $4.99 \times 10^{-9}$ & $1.33 \times 10^{-5}$ \\
\hline$P d p 2$ & Pyruvate dehydrogenase phosphatase catalytic subunit 2 & 1.02102 & 0.00013 & 0.02283 \\
\hline Slc7alo & $\begin{array}{l}\text { Solute carrier family } 7 \text { (cationic amino acid transporter, } \\
\text { y+ system) member } 10\end{array}$ & 3.84436 & 0.00020 & 0.02922 \\
\hline$U c p 3$ & Uncoupling protein 3 & 2.20918 & $2.18 \times 10^{-10}$ & $1.01 \times 10^{-6}$ \\
\hline Zbtb16 & Zinc finger and BTB domain containing 16 & 1.03085 & $3.44 \times 10^{-6}$ & 0.00162 \\
\hline
\end{tabular}

Table III. Top 14 downregulated differentially expressed genes.

\begin{tabular}{|c|c|c|c|c|}
\hline Symbol & Description & log2 Fold-change & P-value & padj \\
\hline Ahsg & $\alpha$-2-HS-glycoprotein & -10.24080 & $1.09 \times 10^{-6}$ & 0.00070 \\
\hline Apoal & Apolipoprotein A-I & -11.97320 & $5.62 \times 10^{-11}$ & $5.23 \times 10^{-7}$ \\
\hline Apoa2 & Apolipoprotein A-II & -9.40264 & $2.80 \times 10^{-12}$ & $5.21 \times 10^{-8}$ \\
\hline Apoc3 & Apolipoprotein C-III & -8.84633 & $1.61 \times 10^{-6}$ & 0.00098 \\
\hline Cyp3a11 & Cytochrome P450 family 3 subfamily a polypeptide 11 & -9.59534 & $8.70 \times 10^{-8}$ & 0.00012 \\
\hline Fabpl & Fatty acid binding protein 1 & -11.30380 & $3.29 \times 10^{-5}$ & 0.00867 \\
\hline$F g b$ & Fibrinogen $\beta$ chain & -10.26910 & $1.55 \times 10^{-5}$ & 0.00487 \\
\hline Matla & Methionine adenosyltransferase I $\alpha$ & -11.72980 & $9.67 \times 10^{-8}$ & 0.00013 \\
\hline Mup3 & Major urinary protein 3 & -9.80745 & $2.20 \times 10^{-5}$ & 0.00628 \\
\hline Serpinalb & Serine (or cysteine) peptidase inhibitor clade A member 1B & -10.98830 & $8.24 \times 10^{-6}$ & 0.00301 \\
\hline Serpinald & Serine (or cysteine) peptidase inhibitor clade A member 1D & -9.29357 & 0.00012 & 0.02139 \\
\hline Serpinale & Serine (or cysteine) peptidase inhibitor clade A member $1 \mathrm{E}$ & -10.98070 & 0.00010 & 0.02026 \\
\hline Serpina $3 k$ & Serine (or cysteine) peptidase inhibitor clade A member $3 \mathrm{~K}$ & -12.06030 & $3.31 \times 10^{-5}$ & 0.00867 \\
\hline Slcolb2 & Solute carrier organic anion transporter family member $1 \mathrm{~b} 2$ & -10.17570 & $7.99 \times 10^{-5}$ & 0.01651 \\
\hline
\end{tabular}

and UCP3 protein expression levels (Fig. 8A and B), as well as reduced mRNA expression of Sirt1, Pgcl $\alpha, N r f 1$, Tfam and Ucp 2 compared with the young adult group (Fig. 8C and D). Moreover, PGC1 $\alpha$ was increased in ATG-125 treated young adult mice challenged with sucrose as well as $P g c l \alpha, N r f 1$ and Tfam expression levels. These results therefore indicated that the mitochondrial biogenesis may be replenished in sucrose-induced mice.

ATG-125 enhances IGF-AKT-mTOR phosphorylation pathways during muscle atrophy in young adult mice.
To identify the role of ATG-125 on protein synthesis via regulation of insulin and mTOR signaling in sucrose-induced muscle atrophy, the activity of the IGF-AKT-mTOR phosphorylation pathway in gastrocnemius muscle was assessed. p-IGF1R, p-IRS1, p-PI3K, p-AKT, p-mTOR, p-S6K and p-4EBP1 protein levels and Igflr, Irsl, Pi3k, Akt, mtor, S6k and $4 e b p 1$ mRNA expression levels were significantly reduced in the sucrose-induced young adult mice compared with the ATG-125 treated mice, and ATG-125 increased the phosphorylation of members of the IGF-AKT-mTOR pathway compared with the sucrose-induced mice, suggesting 
A

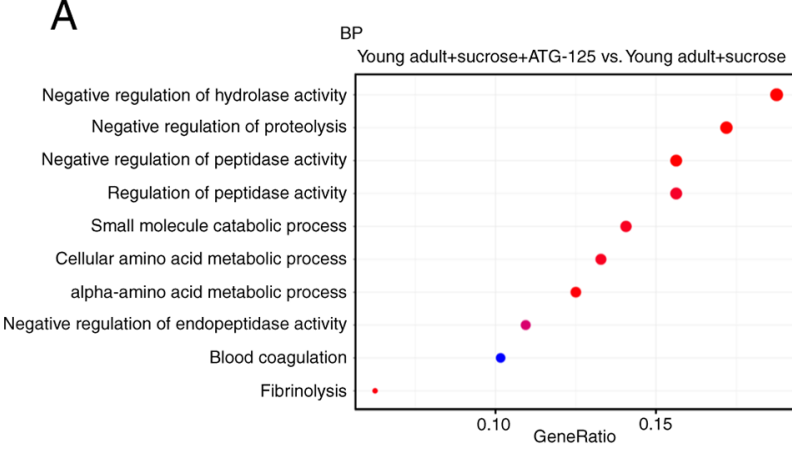

C

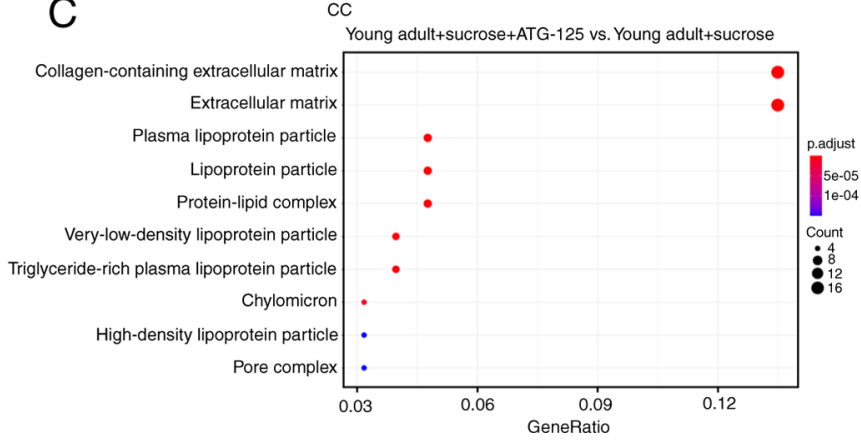

B

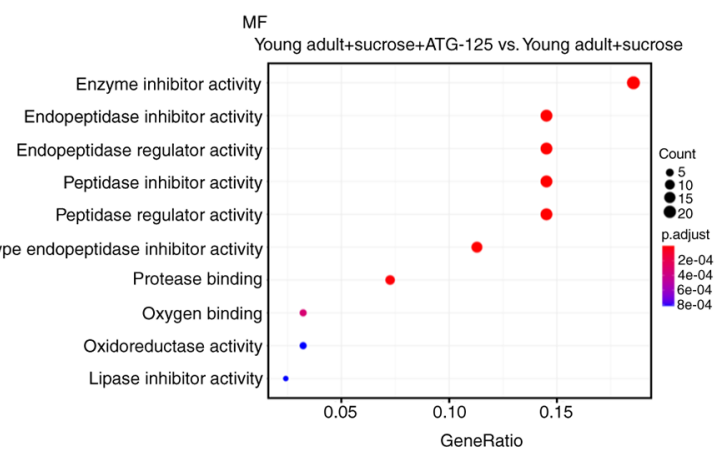

$\mathrm{D}$

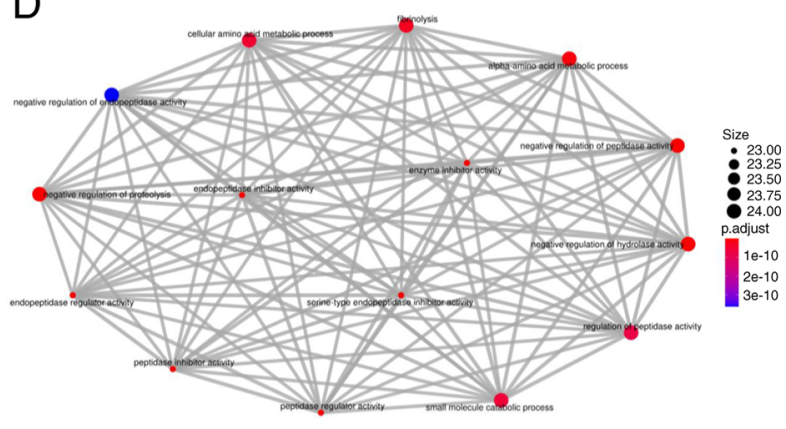

Figure 3. Enrichment GO plot showing the association between the top 10 most significantly enriched GO terms, by grouping similar terms together. The primary (A) BP, (B) MF and (C) CC of differentially expressed genes between sucrose-induced muscle atrophy mice and ATG-125 treated sucrose-induced young adult muscle atrophy mice. (D) Category emapplot shows the associations between the genes associated with the top 15 most significant GO terms and the fold changes of the significant genes associated with these terms. The size of the GO terms reflects the P-values of the term, with the more significant terms being larger. In the enrichment plot, the color represents the P-values relative to the other displayed terms (brighter red is more significant) and the size of the terms represents the number of genes. GO, Gene Ontology; BP, biological process; MF, molecular function; CC, cellular component.

increased activity of this pathway in the gastrocnemius muscle (Fig. 9A and B). These data suggested that ATG-125 promoted protein synthesis by enhancing IGF-AKT-mTOR pathway activity in sucrose-induced muscle atrophy.

\section{Discussion}

Several studies have analyzed the effects of sugar additives on metabolic syndromes in humans as well as in animal models (92-94). The hypothesis of the present study was that as sugar additives can induce inflammation, oxidative stress and IR, this could in turn accelerate muscle atrophy. Sugar-induced metabolic disturbances are reduced by polyphenols/antioxidants, such as resveratrol (92), quercetin (93) and rutin (94), supplementation of which restores muscle protein synthesis sensitivity in older rats. Thus, it was proposed that ATG-125 may also be efficient in counteracting the hypothetical accelerating effect sugar additives have on muscle atrophy; thus, the progression of muscle weight, markers of inflammation and IGF/AKT/mTOR pathways of muscle protein synthesis were assessed.

A comprehensive understanding of the effects of ATG-125 on sucrose-induced young adult muscle atrophy was performed in the present study, thus the network of genes involved in this modulation was determined to illustrate the potential advantage of ATG-125, with the aim of identifying the core candidate genes and pathways involved using a sucrose-induced muscle atrophy mouse model treated with ATG-125. The DEGs were filtered using R and GO and KEGG pathway analysis were performed. First, DEGs (18 upregulated and 14 downregulated) were identified, followed by GO analysis, which revealed that the majority of the DEGs were significantly enriched in 'negative regulation of proteolysis', 'negative regulation of peptidase activity', 'cellular amino acid metabolic process', 'small molecule catabolic process', 'blood coagulation' and 'fibrinolysis' in the BP category. Signaling pathway analysis indicated that the DEGs shared common pathways in 'biosynthesis of amino acids', 'fatty acid elongation', 'cholesterol metabolism', and 'cell cycle'.

The presence of Serpina genes as a DEG facilitates the identification of potential underlying mechanisms involved in disease occurrence/progression. In the present study, Serpina 3 was downregulated in the mouse model of muscle atrophy treated with ATG-125 in DO and KEGG pathway enrichment analyses. The DO results showed that Serpina 3 was significantly enriched in vascular diseases, blood coagulation disorders, deep vein thrombosis, ischemic stroke and age-related macular degeneration pathways. Therefore, it was hypothesized that SERPINA3 may play an important role in high sucrose intake via regulation of signaling pathways involved in muscle oxidative stress and inflammation. Indeed, SERPINA3 is involved in several complex vascular diseases, where it is always involved in inflammation, activating thrombin biological processes in various biological networks (95). SERPINA3, also known as al-antichymotrypsin, is an acute phase protein, the levels of which increase in acute and chronic inflammation (95).

Increasing evidence points to extensive crosstalk between pro-inflammatory cytokines and other mediators that lead to activation of the coagulation system and downregulation of important physiological anticoagulant pathways (96-98), 


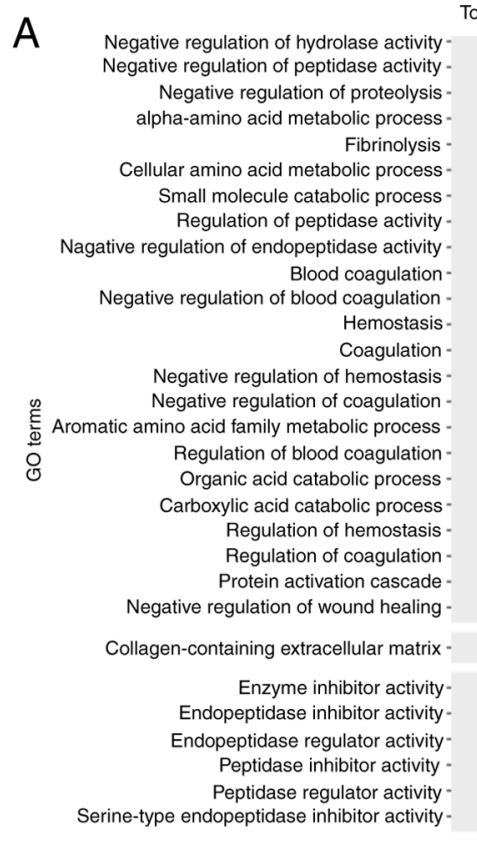

Top 30 ALL GO enrichment : UP vs. Down gene counts
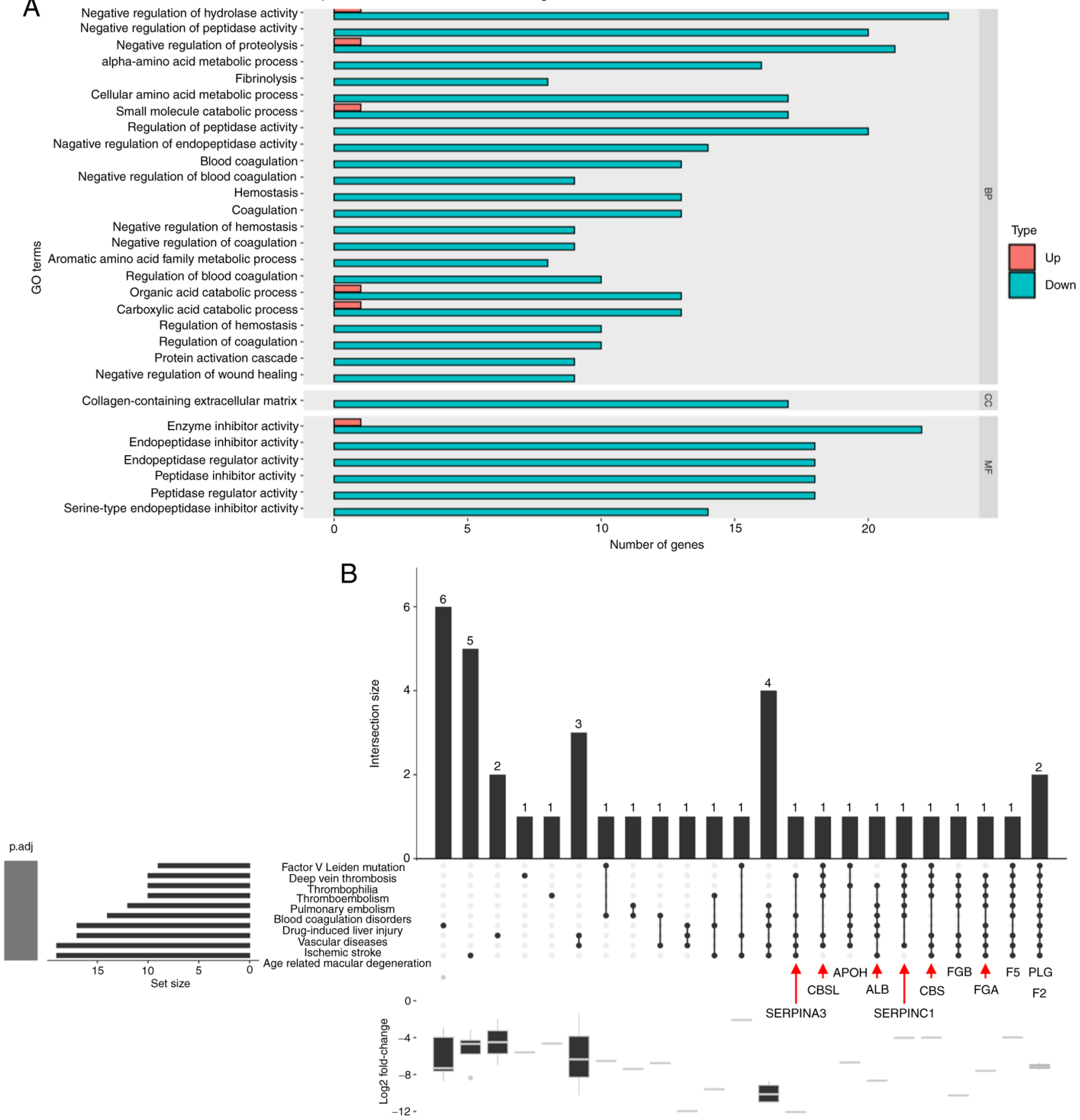

Figure 4. GO enrichment and DO analysis of ATG-125-regulated differentially expressed genes. (A) GO graph providing a summary of the top 30 enriched GO terms. Different colors represent different GO categories. Red and blue represent up- and downregulated genes, respectively. (B) Graph providing summary of the top 10 enriched DO items. GO, Gene Ontology; DO, Disease Ontology.

and coagulation also considerably affects inflammatory activity (98). The expression levels of SERPINA3 in thick choroids was found to be higher in the eyes of patients with early age-related macular degeneration (99), in which SERPINA3 expression may increase oxidative stress and inflammation, a leading cause of blindness, highlighting its potential as a biomarker of macular degeneration. In the present study, Serpina3 was one of the top 30 DEGs and was significantly downregulated in the ATG-125 treated mice. Therefore, it was hypothesized that ATG-125 may play a protective role in the aforementioned vascular-associated diseases when challenged with high sucrose levels.
In sucrose challenged mice, associations between the abundance of mRNA transcripts associated with mitochondrial biogenesis, such as Nmrk2, Ucp3, Slc7a10 (a cationic amino acid transporter) and pyruvate dehydrogenases (Pdk4 and $P d p 2$ ) pathways were observed, consistent with the potential protective role of ATG-125 in muscles from metabolic and functional degeneration. Conversely, in the sucrose-induced gastrocnemius muscle atrophy mouse model, it was observed that ATG-125 significantly downregulated serine (or cysteine) peptidase inhibitor (Serpina $3 k, 1 b, 1 d$ and $1 e$ ) levels, concurrently, all of which are closely associated with specific vascular functions in the DO list, including 'deep vein thrombosis', 

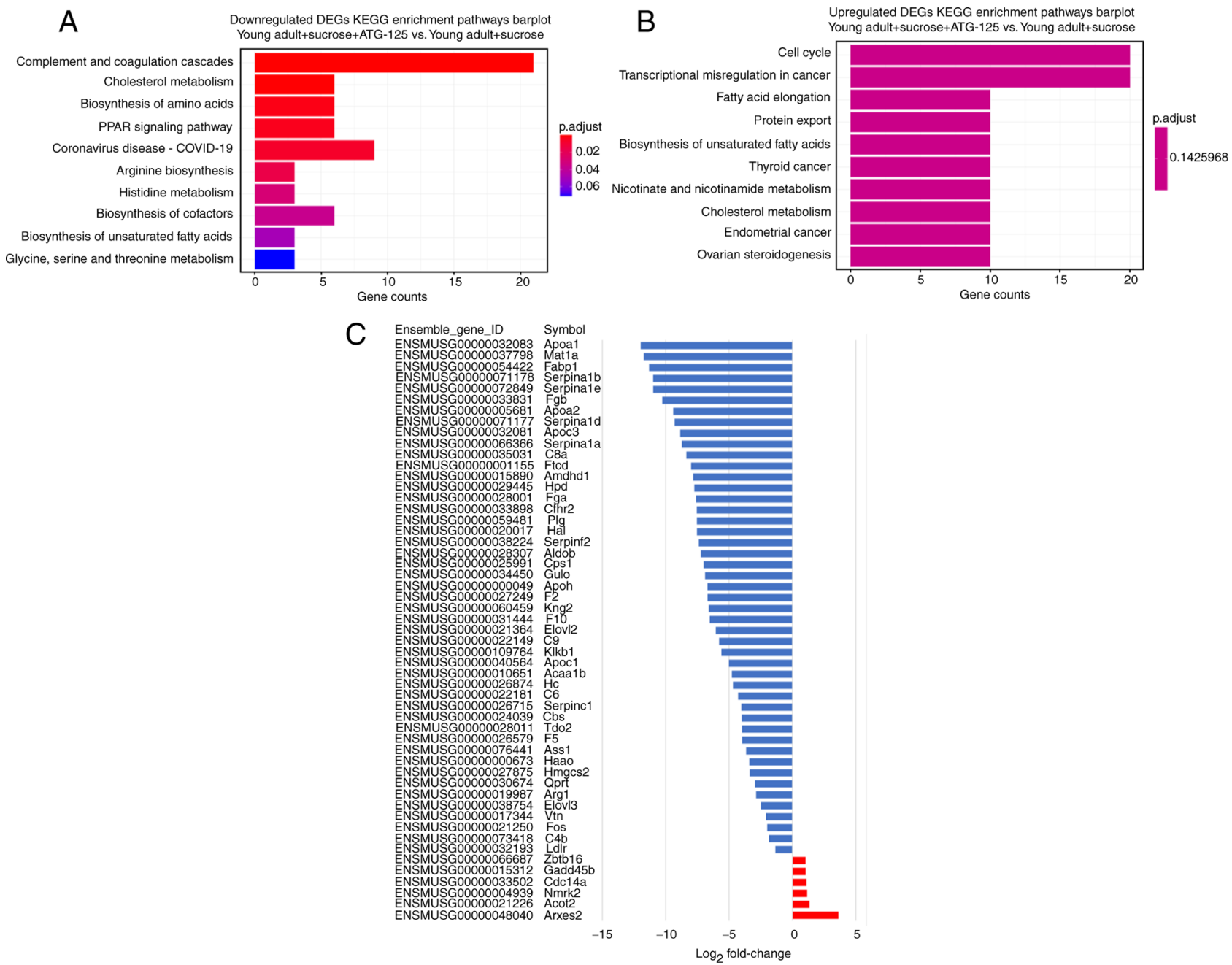

Figure 5. KEGG pathway enrichment analysis of DEGs. Bar plot showing the top 100 (A) downregulated and (B) upregulated KEGG pathways between sucrose-induced muscle atrophy mice and ATG-125 treated sucrose-induced muscle atrophy mice. (C) Bar plot showing genes associated with the top significantly regulated KEGG pathways between sucrose-induced muscle atrophy mice and ATG-125 treated sucrose-induced muscle atrophy mice. KEGG, Kyoto Encyclopedia of Genes and Genomes; DEG, differentially expressed gene.

'blood coagulation disorders', 'vascular diseases', 'ischemic stroke' and 'age-related macular degeneration' based on DO analysis. In addition, ATG-125 reduced the expression of apolipoproteins A-I, A-II and C-III, the major genes involved in high density lipoprotein particle crosstalk with lipid-induced cardiovascular disease risk factors and FFAs, together stimulating inflammation (100-102). Furthermore, the gastrocnemius muscle transcriptomes in the mouse muscular dystrophy model were enriched for various amino acid metabolic processes, lipoprotein particles and protein-lipid complex metabolism-related genes, a number of which were modulated by ATG-125, and were involved in proteolytic events, including inflammation.

Replenishing mitochondrial biogenesis with daily ATG-125 intervention increased the activity of improved muscle IGF-AKT-mTOR, as determined by the increased phosphorylation of its members, in sucrose-induced young adult mice, and reversed the NF- $\kappa \mathrm{B}-\mathrm{HIF}-1 \alpha-\mathrm{TGF} \beta \mathrm{RII}$ inflammatory cascade in the model of muscle atrophy. The effects of mitochondrial energy repletion in the sucrose-induced mouse model relied on the improvement in mitochondrial function and on the reduction in general proteolysis, inflammation and degradation.
In summary, the results of the present study suggested that the administration of ATG-125 may benefit mice with muscular dystrophies or other neuromuscular degenerative conditions characterized by the aforementioned gene expression signatures. Mitochondria play a crucial role in the maintenance of ionic balance and other organelles as a source of energy. PGC- $1 \alpha$ plays a crucial role in regulating mitochondrial biogenesis and function, particularly in skeletal muscle (103). SIRT1 modulates PGC-1 $\alpha$ expression and activity (104), which regulates mitochondrial biogenesis and function through the induction and activation of several downstream nuclear transcription factors (including TFAM and NRF1) $(104,105)$. In the present study, the results showed that ATG-125 significantly increased the PGC-1 $\alpha$, SIRT1 protein and Nrf1 and Tfam mRNA expression levels and the mitochondrial UCP1, 2 and 3 levels, suggesting that ATG-125 played a crucial role in regulating mitochondrial biogenesis and function. Moreover, studies have demonstrated that enhanced mitochondrial biogenesis and function may play a primary role in elevated type I muscle fiber formation and may be a novel mediator of skeletal muscle fiber type $(106,107)$. Although the present study did not determine whether type I muscle fibers were 


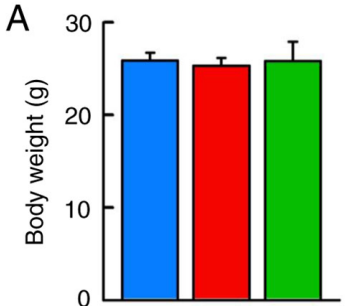

$\mathrm{B}$

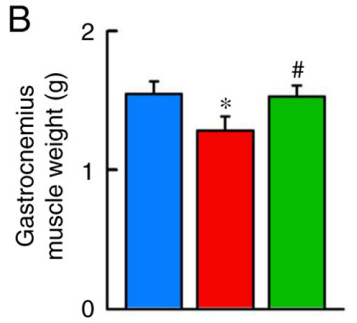

C

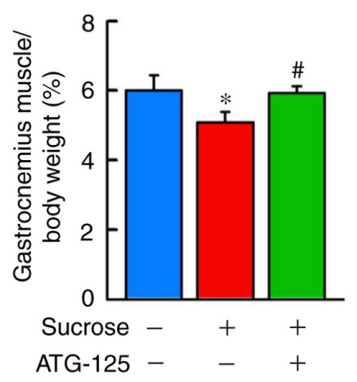

$E$
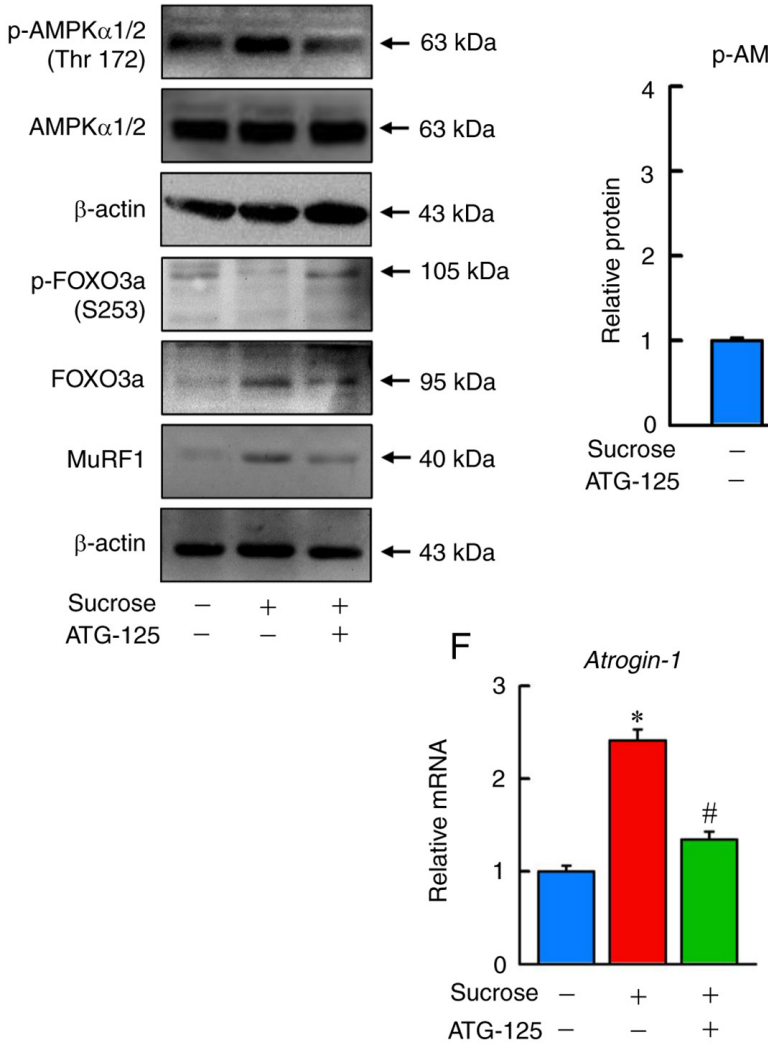

D
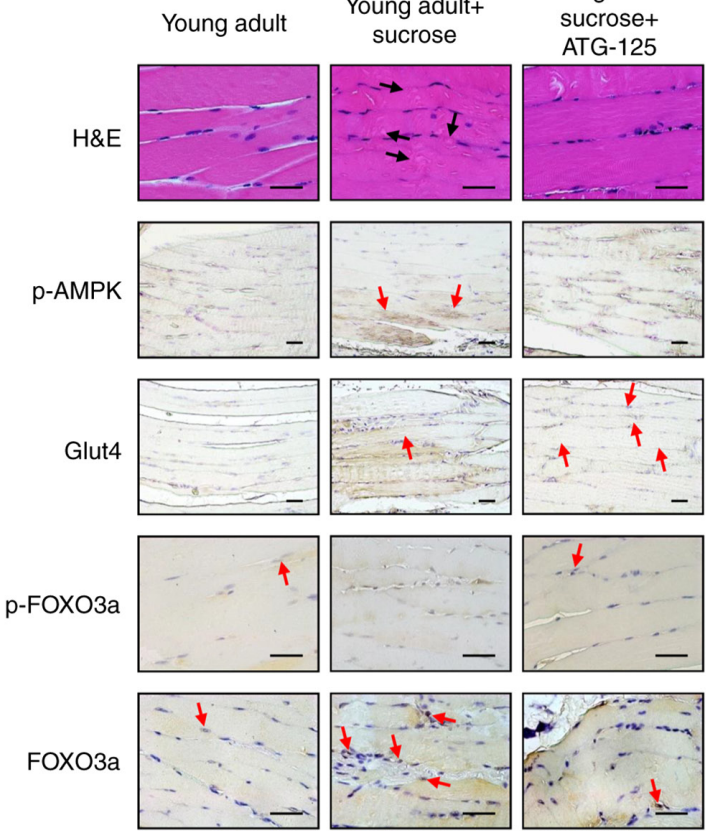

MuRF1
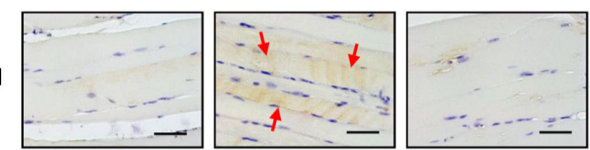
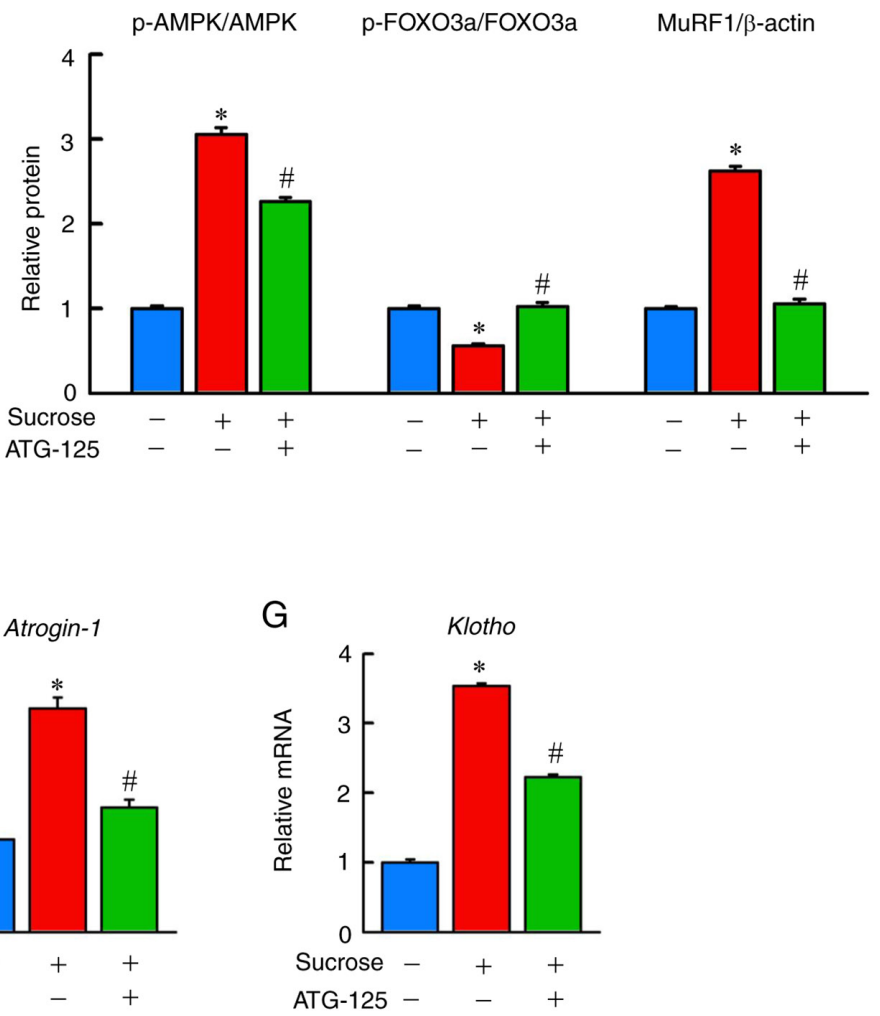

Figure 6. ATG-125 improves sucrose-induced muscle atrophy in mice. The (A) body weight, (B) muscle weight and (C) gastrocnemius muscle/body weight ratio between the young adult mice, sucrose-induced muscle atrophy mice and ATG-125 treated sucrose-induced muscle atrophy mice. (D) Representative H\&E staining and immunohistochemical analysis of p-AMPK, Glut4, p-FOXO3a, FOXO3a and MuRF1 in muscle sections of the young adult mice, sucrose-induced muscle atrophy mice and ATG-125 treated sucrose-induced muscle atrophy mice. Red arrows highlight the positive staining. Scale bar, $100 \mu \mathrm{m}$. (E) p-AMPK, AMPK, p-FOXO3a, FOXO3a and MuRF1 protein expression levels were determined by western blotting. (F and G) Atrogin-1 and Klotho mRNA expression levels were determined by reverse transcription-quantitative PCR relative to Gapdh. Data are presented as the mean \pm standard error of the mean of five independent experiments. " $\mathrm{P}<0.05$ vs. Young adult mice; ${ }^{*} \mathrm{P}<0.05$ vs. Sucrose-induced muscle atrophy mice. H\&E, hematoxylin and eosin; $\mathrm{p}$-, phosphorylated; AMPK, AMP-activated protein kinase; FOXO3a, forkhead box O3a; Glut4, glucose transporter type 4 insulin-responsive; MuRF1, E3 ubiquitin-protein ligase TRIM63. 


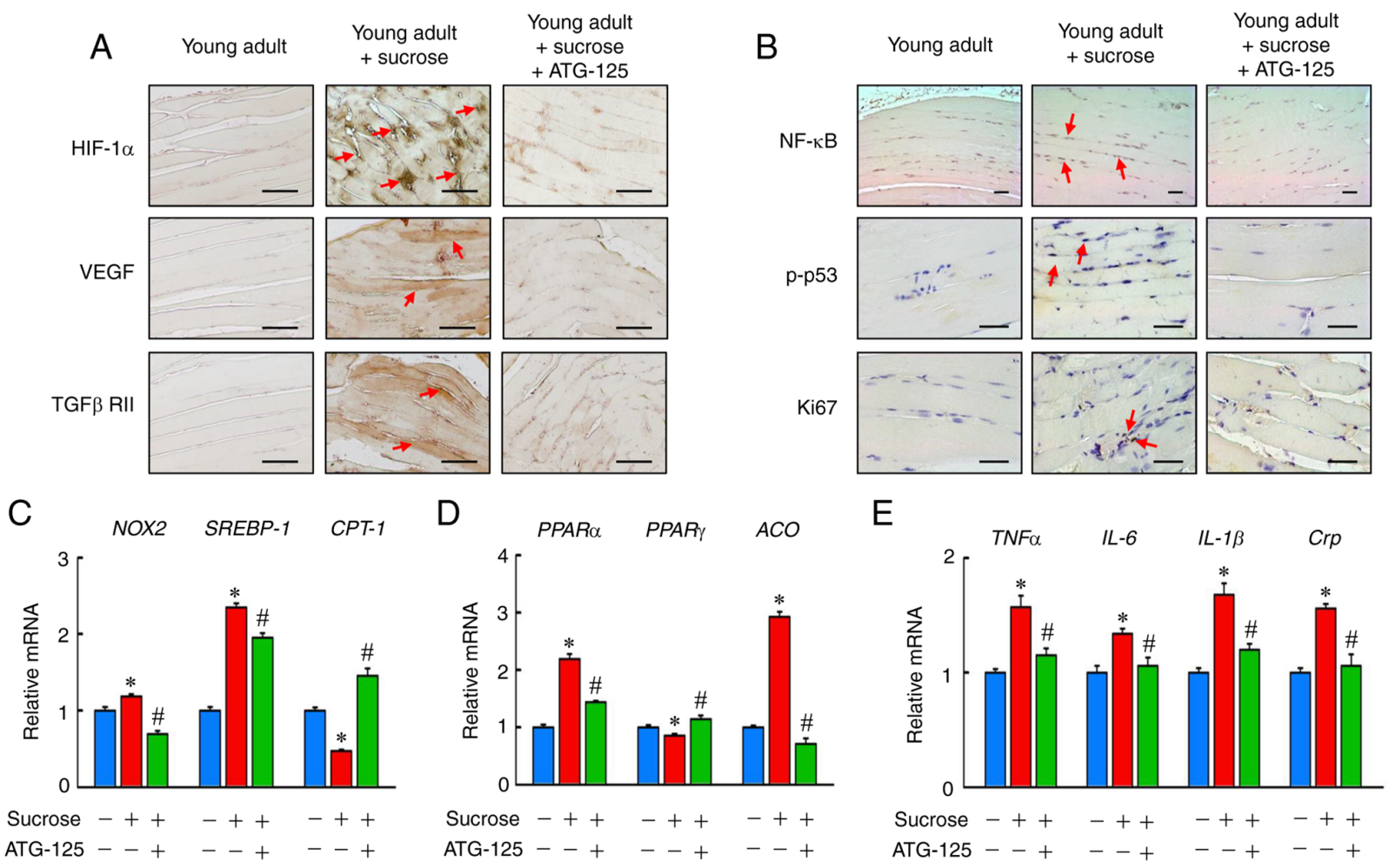

Figure 7. ATG-125 improves sucrose-induced inflammation in mice muscle atrophy. (A) Immunohistochemistry analysis of HIF-1 $\alpha$, VEGF, TGF $\beta R I I$, (B) NF-кB, p-p53 and Ki67 staining of the muscle sections. Red arrows highlight the positive staining. Scale bar, $100 \mu \mathrm{m}$. (C) Nox2, Srebp-1, Cpt-1, (D) Ppara, Ppar $\gamma$, Aco, (E) Tnfa, Il-6, Il-1 $\beta$ and Crp mRNA expression levels were determined by reverse transcription-quantitative PCR. Data are presented as the mean \pm the standard error of the mean of five independent repeats. ${ }^{*} \mathrm{P}<0.05$ vs. Young adult mice; ${ }^{\prime \prime} \mathrm{P}<0.05$ vs. Sucrose-induced muscle atrophy mice. HIF- $1 \alpha$,

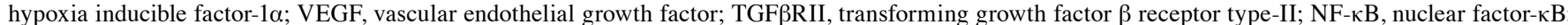
p-, phosphorylated; Nox2, NADPH oxidase 2; Srebp-1, sterol regulatory element-binding protein 1; Cpt-1, carnitine palmitoyltransferase 1; Ppar, peroxisome proliferator-activated receptor; Aco, acyl-CoA oxidase; Tnf $\alpha$, tumor necrosis factor $\alpha$; Il-6, interleukin-6; Crp, C-reactive protein.

\section{A}
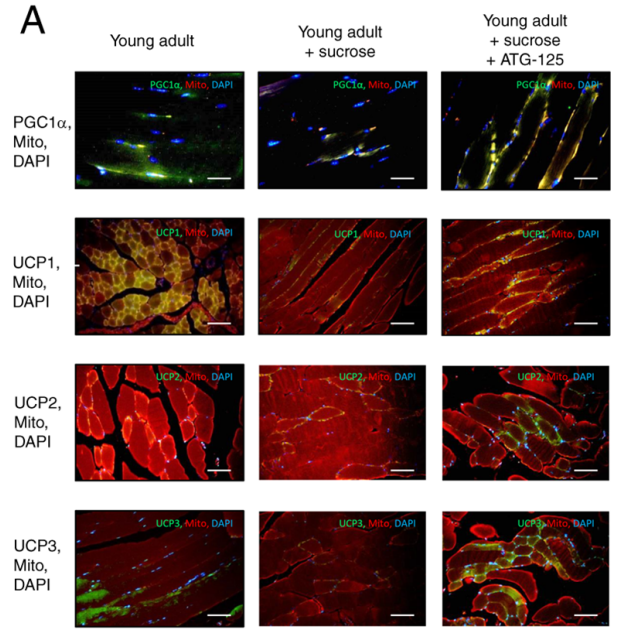

C
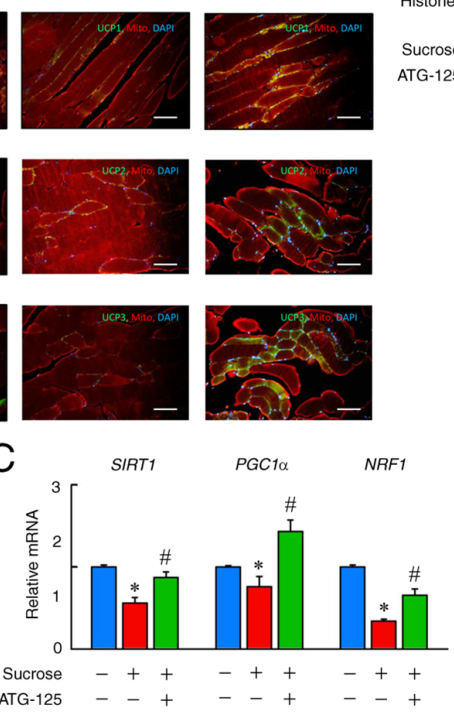
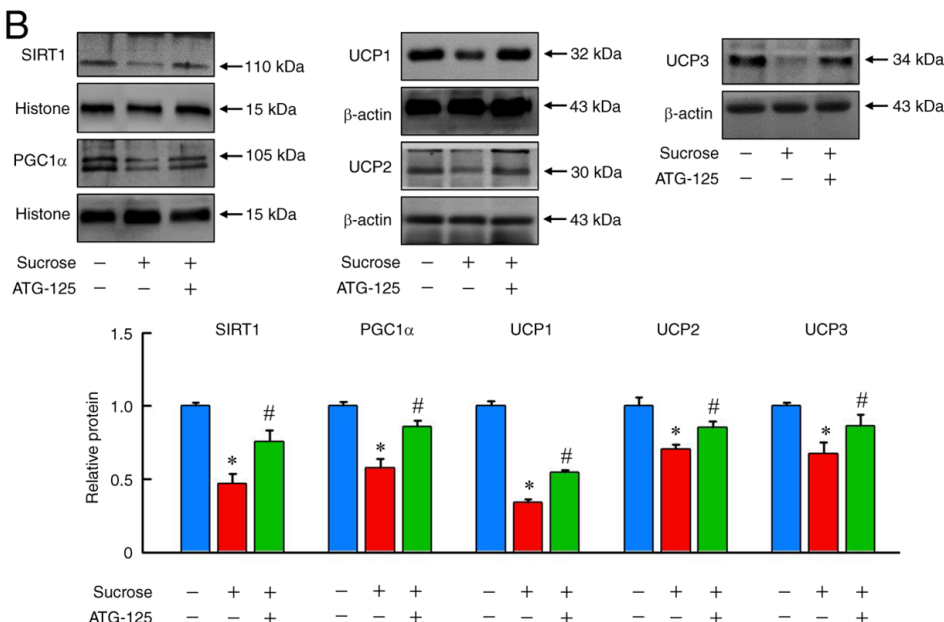

ATG-125

$\mathrm{D}$

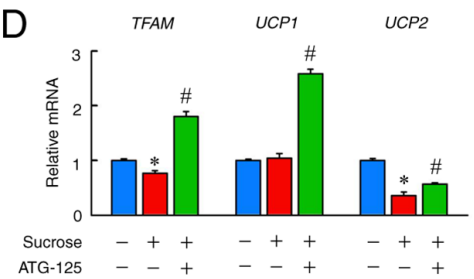

Figure 8. ATG-125 improves sucrose-induced mitochondrial dysfunction in mice muscle atrophy. (A) Immunofluorescence analysis of PGC1 $\alpha$, UCP1, UCP2 and UCP3 expression, as well as Mito tracker staining for muscle sections. Scale bar, $100 \mu \mathrm{m}$. (B) SIRT1, PGC1 $\alpha$, UCP1, UCP2 and UCP3 protein expression levels were determined by western blotting. (C) Sirt1, Pgcla, Nrf1, (D) Tfam, Ucp1 and Ucp 2 mRNA expression levels were determined by reverse transcription-quantitative PCR. Data are presented as the mean \pm the standard error of the mean of five independent repeats. ${ }^{*} \mathrm{P}<0.05$ vs. Young adult mice; ${ }^{\#} \mathrm{P}<0.05$ vs. Sucrose-induced muscle atrophy mice. SIRT1, sirtuin-1; PGC-1, peroxisome proliferator-activated receptor $\gamma$ coactivator 1- $\alpha$; Nrf1, nuclear respiratory factor 1; Tfam, mitochondrial transcription factor A; Ucpl, uncoupling protein 1. 
A
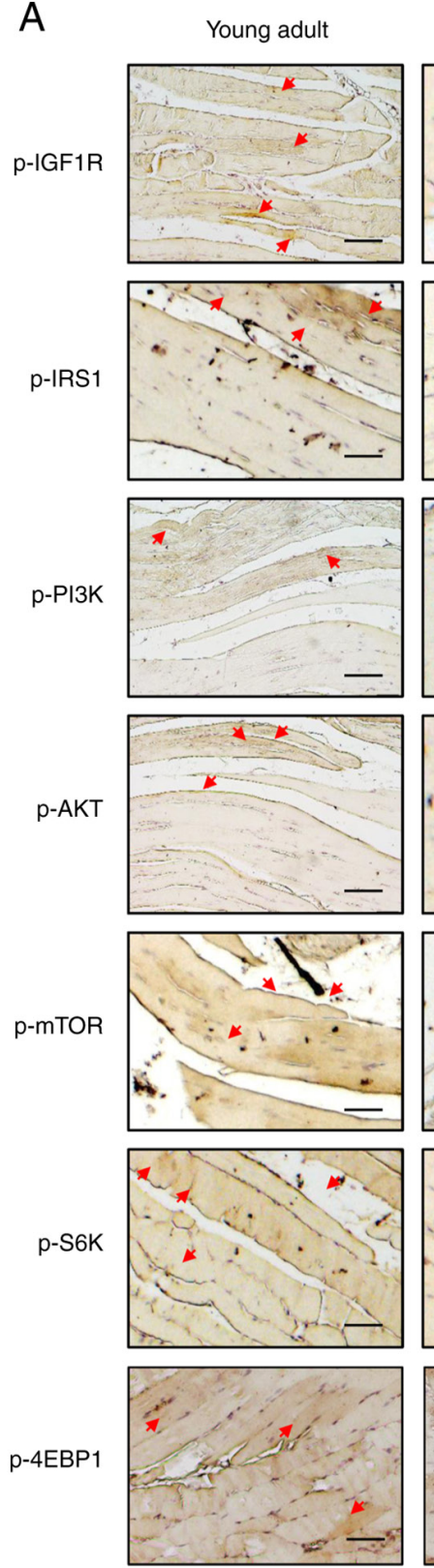

Young adult+sucrose
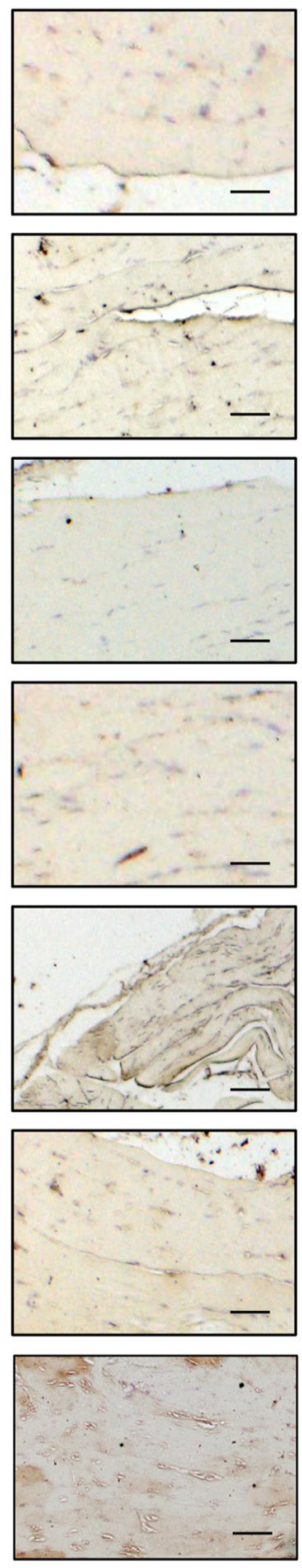

Young adult+sucrose+ ATG-125
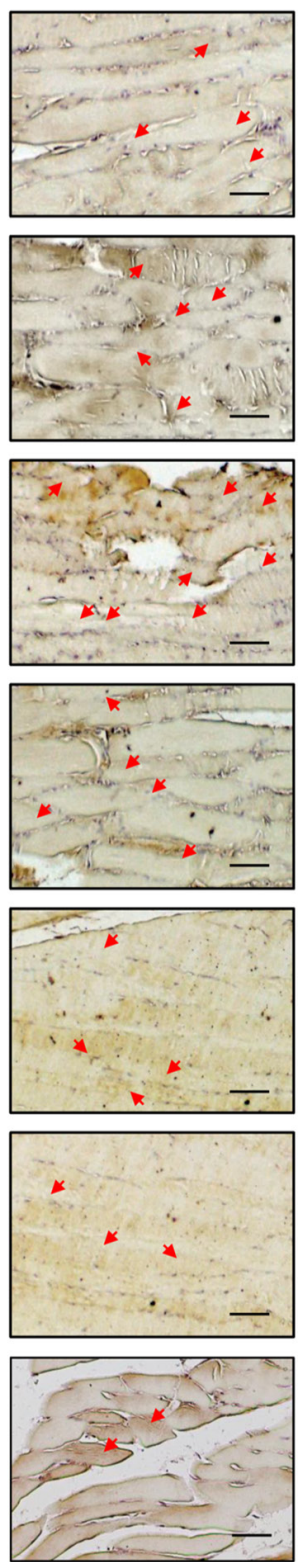

B

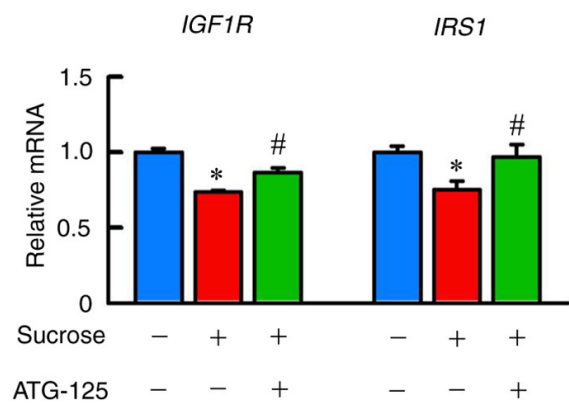

PI3K

$A K T$

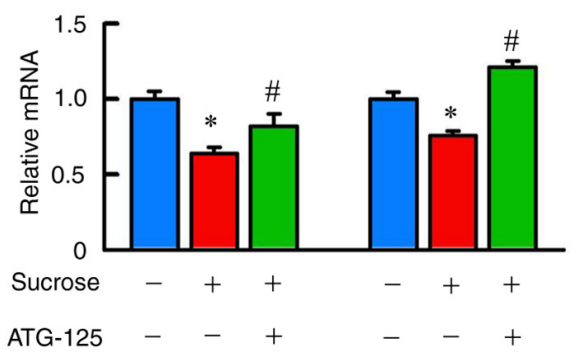

mTOR

S6K

4EBP1

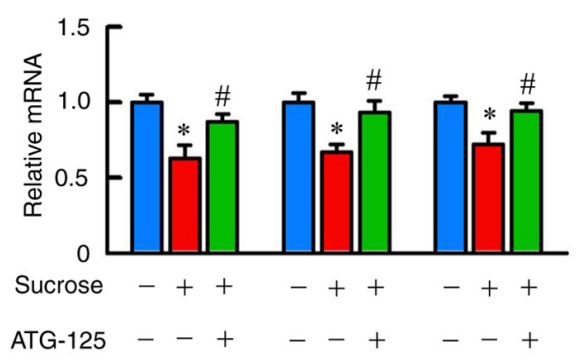

Figure 9. ATG-125 reverses the impaired sucrose-induced insulin signaling in muscle atrophy in young adult mice. (A) Immunohistochemical analysis of p-IGF1R, p-IRS1, p-PI3K, p-AKT, p-mTOR, p-S6K and p-4EBP1 staining in muscle sections are shown. Red arrows highlight the positive staining. Scale bar, $100 \mu \mathrm{m}$. (B) Igflr, Irs 1, Pi3k, Akt, mtor, S6k and 4ebpl mRNA expression levels were determined by reverse transcription-quantitative PCR. Data are presented as the mean \pm the standard error of the mean of five independent repeats. ${ }^{*} \mathrm{P}<0.05$ vs. Young adult mice; ${ }^{\sharp} \mathrm{P}<0.05$ vs. Sucrose-induced muscle atrophy mice. $\mathrm{p}-$, phosphorylated; IGF1R, insulin-like growth factor 1 receptor; IRS1, insulin receptor substrate 1; PI3K, phospho-inositide 3-kinase; AKT, protein kinase B; mTOR, mechanistic target of rapamycin; S6K, ribosomal protein S6 kinase; 4EBP1, eukaryotic translation initiation factor 4E-binding protein 1.

more abundant in the ATG-125 treated mice, it was shown that ATG-125 downregulated the mRNA expression levels of type I muscle fiber-related genes (Tnnil, Tnncl and Tnntl) in the sucrose-challenged gastrocnemius muscle. The transcriptomic data suggested that, at least in part, ATG-125 may promote the formation of type I muscle fibers in mice muscles, which is dependent on the improvement of mitochondrial biogenesis.

Indeed, PGC- $1 \alpha$ plays a crucial role in the control of mitochondrial biogenesis, which is intricately involved in core muscle physiology and exercise physiology through its interaction with FOXO and NF-kB and its regulation by the SIRT pathway and apoptotic cascades (108-110). The activation of FOXO3 enhances atrogin-1 and MuRF-1 transcriptional activity $(111,112)$. Furthermore, NF- $\kappa$ B activation promotes pro-inflammatory cytokine and myokine production, such as IL-1 $\beta$, IL- 6 , TNF- $\alpha$ and monocyte chemoattractant protein $(113,114)$. Following ATG-125 treatment, there was a reduction in $\mathrm{NF}-\kappa \mathrm{B}$ expression, a known deacetylation target of SIRT1, suggesting that a decrease in mitochondria-dependent SIRT1 activity was involved in the improved inflammatory state of the skeletal muscle in the sucrose-challenged mice. In the muscle, myofibrillar proteins serve as the major amino acid reservoir in the organism, therefore, protein degradation serves a physiological role distinct from proteolysis in other tissues. While proteasomes degrade myofibrillar and most soluble proteins (115), organelles 
(especially the mitochondria) are degraded primarily in lysosomes (116). Although atrogin-1 and MuRF1 proteolytic pathways are activated by FOXO3, and this results in reduced strength due to loss of myofibrillar components, as well as in reduced endurance due to loss of the mitochondria, it presumably ensures that the loss of different cell components is coordinated upon fasting or disuse, allowing the muscle to maintain a relatively normal composition. This difference may explain why proteasome-dependent proteolysis accounts for only a small fraction of the FOXO3-induced proteolysis in myotubes, whereas this system accounts for the majority of the accelerated proteolysis in normal and atrophying adult muscles (117). The analysis performed in the present study, combining unbiased transcriptomics and biomedical analysis approaches, identified an important mechanism for protecting the muscle from wasting during excessive sucrose intake. IGF1-AKT-mTOR phosphorylation was strongly downregulated by sucrose, which led to loss of muscle mass by concurrently activating proteolysis and disrupting homeostatic muscle growth. ATG-125 may exert its protective effects by regulating the balance between distinct growth-associated mTOR complexes, which strongly inhibit IGF1-AKT-mTOR phosphorylation, while supporting mitochondrial biogenesis. Various physiological or pathological hypertrophic growth stimuli lead to activation of AKT by phosphorylation, which causes inactivation of FOXO factors via phosphorylation and nuclear exclusion, thus limiting the expression of atrophy-related genes (118). The present study demonstrated that sucrose inactivates phosphorylation of the PI3K-AKT pathway, thus IGF-1-stimulated protein synthesis is inhibited, resulting in degradation of myofibrillar proteins and expression of Atrogen-1 and MuRF1. The ATG-125-induced inhibition of proteolysis appears to contribute to muscle growth via inactivation of FOXO3 through AKT phosphorylation, thus maintaining proteostasis and organelle quality for homeostatic preservation of muscle mass.

Loss of muscle mass and force significantly affects the health, quality of life and even survival of an individual. Given its beneficial effects on reducing muscle wasting, ATG-125 should be regarded as a novel regulator of mammalian muscle growth with potentially broad applications in the treatment of common catabolic conditions. However, the present study has certain limitations: i) By mixing all the phytochemicals together, the focused insights in single compound characteristics on muscle atrophy were not assessed. ii) Bioinformatics was only performed in young adult mice and the mechanisms identified may not apply to the muscle atrophy observed in aging mice or type 2 diabetic animals. iii) ATG-125 is a phytochemicals-rich herbal formula that contains multiple antimicrobial and antioxidant properties herbs. In addition to their diverse bioactive properties in animal models, they have potential effects in the maintenance of human health. Due to this relevance, highly-specific qualitative and quantitative methods of analysis are necessary to accurately analyze bioactive substance in different matrices derived from ATG-125. In future studies, a sensitive and specific multiple reaction monitoring or parallel reaction monitoring analysis method is required to detect the verification of screened proteins that may be involved in relation to muscle atrophy signaling. iv) This study did not observe the dose-dependent effects of
ATG-125 at high or low doses, which leads to unclear estimates of the acceptable potency of ATG-125. In other words, the current results just raised a 'reference dose' or 'acceptable concentration' of ATG-125. The lack of dose response and positive controls are major limitations of this study. Additional databases should be used for validation of the effects of ATG-125 in future studies.

In conclusion, the results of the present study showed that ATG-125 may exert protective effects against sucrose-induced muscle atrophy via regulation of the AKT/FOXO3 pathways, which regulates proteolysis, as well as by improving mitochondrial dysfunction.

\section{Acknowledgements}

Not applicable.

\section{Funding}

The present study was supported by funding from Chang Gung University (grant nos. SCRPD1J0141, SCRPD1J0221 and SCRPD1K0651).

\section{Availability of data and materials}

The datasets used and/or analyzed during the current study are available from the corresponding author on reasonable request. Sequence Read Archive (SRA; https://www.ncbi.nlm. nih.gov/sra) stores raw DNA sequencing data and alignment information from high-throughput sequencing platforms. BioSample (accession no. SAMN22871017) records are indexed and searchable. The datasets generated and/or analyzed during the current study are available in the BioProjects (accession no. PRJNA777698) repository (https://www.ncbi.nlm.nih. gov/bioproject/?term=PRJNA777698), as well as with derived experimental data in NCBI's primary archives, including SRA (accession no. SRR16767413-SRR16767421), which all identify genes from muscle tissue samples.

\section{Authors' contributions}

CCY, HML, HHC and TYL designed the experiments. CCY, HML, MCL, YLL and TYL performed the experiments. CCY and HML analyzed the data. CCY, HML, HHC and TYL wrote the manuscript. All authors have read and approved the final manuscript. WHC supervised the study and interpreted the data. MCL and YLL confirm the authenticity of all the raw data.

\section{Ethics approval and consent to participate}

All animal experiments were performed in accordance with the Guide for the Care and Use of Laboratory Animals and were approved by the Chang Gung University Animal Care and Use Committee (approval no. CGU108-111; Taoyuan, Taiwan).

\section{Patient consent for publication}

Not applicable. 


\section{Competing interests}

The authors declare that they have no competing interests.

\section{References}

1. Lynch GS, Schertzer JD and Ryall JG: Therapeutic approaches for muscle wasting disorders. Pharmacol Ther 113: 461-487, 2007.

2. Bonaldo P and Sandri M: Cellular and molecular mechanisms of muscle atrophy. Dis Model Mech 6: 25-39, 2013.

3. Ferrucci L and Guralnik JM: Inflammation, hormones, and body composition at a crossroad. Am J Med 115: 501-502, 2003.

4. Pedersen BK and Bruunsgaard $\mathrm{H}$ : Possible beneficial role of exercise in modulating low-grade inflammation in the elderly. Scand J Med Sci Sports 13: 56-62, 2003.

5. Franceschi C, Bonafè M, Valensin S, Olivieri F, De Luca M, Ottaviani E and De Benedictis G: Inflamm-aging. An evolutionary perspective on immunosenescence. Ann N Y Acad Sci 908: 244-254, 2000.

6. Ali S and Garcia JM: Sarcopenia, cachexia and aging: Diagnosis, mechanisms and therapeutic options-a mini-review. Gerontology 60: 294-305, 2014.

7. Costamagna D, Costelli P, Sampaolesi M and Penna F: Role of Inflammation in muscle homeostasis and myogenesis. Mediators Inflamm 2015: 805172, 2015

8. Milan G, Romanello V, Pescatore F, Armani A, Paik JH, Frasson L, Seydel A, Zhao J, Abraham R, Goldberg AL, et al: Regulation of autophagy and the ubiquitin-proteasome system by the FoxO transcriptional network during muscle atrophy. Nat Commun 6: 6670, 2015.

9. Gregor MF and Hotamisligil GS: Inflammatory mechanisms in obesity. Annu Rev Immunol 29: 415-445, 2011.

10. Glass CK and Olefsky JM: Inflammation and lipid signaling in the etiology of insulin resistance. Cell Metab 15: 635-645, 2012.

11. Olefsky JM and Glass CK: Macrophages, inflammation, and insulin resistance. Annu Rev Physiol 72: 219-246, 2010.

12. Perry BD, Caldow MK, Brennan-Speranza TC, Sbaraglia M, Jerums G, Garnham A, Wong C, Levinger P, Asrar Ul Haq M, Hare DL, et al: Muscle atrophy in patients with Type 2 Diabetes Mellitus: Roles of inflammatory pathways, physical activity and exercise. Exerc Immunol Rev 22: 94-109, 2016.

13. Maliszewska K, Adamska-Patruno E and Krętowski A: The interplay between muscle mass decline, obesity, and type 2 diabetes. Pol Arch Intern Med 129: 809-816, 2019.

14. Monier S, Le Cam A and Le Marchand-Brustel Y: Insulin and insulin-like growth factor I. Effects on protein synthesis in isolated muscles from lean and goldthioglucose-obese mice. Diabetes 32: 392-397, 1983

15. Rommel C, Bodine SC, Clarke BA, Rossman R, Nunez L, Stitt TN Yancopoulos GD and Glass DJ: Mediation of IGF-1-induced skeletal myotube hypertrophy by $\mathrm{PI}(3) \mathrm{K} / \mathrm{Akt} / \mathrm{mTOR}$ and $\mathrm{PI}(3)$ K/Akt/GSK3 pathways. Nat Cell Biol 3: 1009-1013, 2001.

16. Sacheck JM, Ohtsuka A, McLary SC and Goldberg AL: IGF-I stimulates muscle growth by suppressing protein breakdown and expression of atrophy-related ubiquitin ligases, atrogin-1 and MuRF1. Am J Physiol Endocrinol Metab 287: E591-E601, 2004.

17. de Alvaro C, Teruel T, Hernandez R and Lorenzo M: Tumor necrosis factor alpha produces insulin resistance in skeletal muscle by activation of inhibitor kappaB kinase in a p38 MAPK-dependent manner. J Biol Chem 279: 17070-17078, 2004

18. Dogra C, Changotra H, Wedhas N, Qin X, Wergedal JE and Kumar A: TNF-related weak inducer of apoptosis (TWEAK) is a potent skeletal muscle-wasting cytokine. FASEB J 21: 1857-1869, 2007.

19. Hirosumi J, Tuncman G, Chang L, Görgün CZ, Uysal KT, Maeda K, Karin M and Hotamisligil GS: A central role for JNK in obesity and insulin resistance. Nature 420: 333-336, 2002.

20. Peterson JM, Bakkar N and Guttridge DC: NF-kB signaling in skeletal muscle health and disease. Curr Top Dev Biol 96: 85-119, 2011.

21. Törrönen R, Kolehmainen M, Sarkkinen E, Mykkänen H and Niskanen L: Postprandial glucose, insulin, and free fatty acid responses to sucrose consumed with blackcurrants and lingonberries in healthy women. Am J Clin Nutr 96: 527-533, 2012.

22. Abdullah A, Peeters A, de Courten M and Stoelwinder J: The magnitude of association between overweight and obesity and the risk of diabetes: A meta-analysis of prospective cohort studies. Diabetes Res Clin Pract 89: 309-319, 2010.
23. Boden G: Obesity and free fatty acids. Endocrinol Metab Clin North Am 37: 635-646, viii-ix, 2008.

24. K S, Senthilkumar GP, Sankar P and Bobby Z: Attenuation of oxidative stress, inflammation and insulin resistance by allium sativum in fructose-fed male rats. J Clin Diagn Res 7: 1860-1862, 2013.

25. Hwang IS, Ho H, Hoffman BB and Reaven GM: Fructose-induced insulin resistance and hypertension in rats. Hypertension 10: 512-516, 1987.

26. Gatineau E, Savary-Auzeloux I, Migné C, Polakof S, Dardevet D and Mosoni L: Chronic intake of sucrose accelerates sarcopenia in older male rats through alterations in insulin sensitivity and muscle protein synthesis. J Nutr 145: 923-930, 2015

27. Shin MS, Lee J, Lee JW, Park SH, Lee IK, Choi JA, Lee JS and Kang KS: Anti-Inflammatory effect of Artemisia argyi on Ethanol-Induced gastric ulcer: Analytical, in vitro and in vivo studies for the identification of action mechanism and active compounds. Plants (Basel) 10 332, 2021.

28. Gao XH, Zhang SD, Wang LT, Yu L, Zhao XL, Ni HY, Wang YQ, Wang JD, Shan CH and Fu YJ: Anti-Inflammatory effects of neochlorogenic acid extract from mulberry leaf (Morus alba L.) against LPS-Stimulated inflammatory response through mediating the AMPK/Nrf2 signaling pathway in A549 Cells. Molecules 25: 1385, 2020.

29. Hu PF, Sun FF and Qian J: Leonurine exerts Anti-Catabolic and

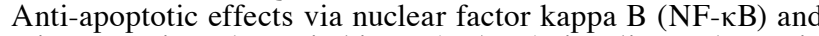
mitogen-activated protein kinase (MAPK) signaling pathways in chondrocytes. Med Sci Monit 25: 6271-6280, 2019.

30. Cho SY, Kim HW, Lee MK, Kim HJ, Kim JB, Choe JS, Lee YM and Jang HH: Antioxidant and Anti-Inflammatory activities in relation to the flavonoids composition of pepper (Capsicum annuum L.). Antioxidants (Basel) 9: 986, 2020.

31. Hernández-Ortega M, Ortiz-Moreno A,Hernández-Navarro MD, Chamorro-Cevallos G, Dorantes-Alvarez L and Necoechea-Mondragón H: Antioxidant, antinociceptive, and anti-inflammatory effects of carotenoids extracted from dried pepper (Capsicum annuum L.). J Biomed Biotechnol 2012: 524019, 2012

32. Lai KH, Chen PJ, Chen CC, Yang SH, El-Shazly M, Chang $\mathrm{YC}, \mathrm{Wu} \mathrm{YH}, \mathrm{Wu} \mathrm{YH}$, Wang $\mathrm{YH}$, Hsieh $\mathrm{HL}$ and Hwang TL: Lophatherum gracile Brongn. attenuates neutrophilic inflammation through inhibition of JNK and calcium. J Ethnopharmacol 264: 113224, 2021.

33. Ti H, Mai Z, Wang Z, Zhang W, Xiao M, Yang Z and Shaw P: Bisabolane-type sesquiterpenoids from Curcuma longa L. exert anti-influenza and anti-inflammatory activities through NF- $\kappa$ B/MAPK and RIG-1/STAT1/2 signaling pathways. Food Funct 12: 6697-6711, 2021.

34. Memarzia A, Khazdair MR, Behrouz S, Gholamnezhad Z, Jafarnezhad M, Saadat S and Boskabady MH: Experimental and clinical reports on anti-inflammatory, antioxidant, and immunomodulatory effects of Curcuma longa and curcumin, an updated and comprehensive review. Biofactors 47: 311-350, 2021.

35. Yue SJ, Qin YF, Kang A, Tao HJ, Zhou GS, Chen YY, Jiang JQ, Tang YP and Duan JA: Total Flavonoids of Glycyrrhiza uralensis Alleviates irinotecan-induced colitis via modification of gut microbiota and fecal metabolism. Front Immunol 12: 628358, 2021.

36. Li M, Chai X, Wang L, Yang J and Wang Y: Study of the variation of phenolic acid and flavonoid content from fresh artemisiae argyi folium to moxa wool. Molecules 24: 4603, 2019.

37. Zhao L, Wang D, Liu J, Yu X, Wang R, Wei Y, Wen C and Ouyang Z: Transcriptomic analysis of key genes involved in chlorogenic acid biosynthetic pathway and characterization of MaHCT from Morus alba L. Protein Expr Purif 156: 25-35, 2019.

38. Chang Y, Zhang D, Yang G, Zheng Y and Guo L: Screening of Anti-Lipase components of Artemisia argyi leaves based on Spectrum-Effect relationships and HPLC-MS/MS. Front Pharmacol 12: 675396, 2021.

39. Wen YQ, Gong LY, Wang L, Zhao N, Sun Q, Kamara MO, Ma HY and Meng FH: Comparative pharmacokinetics study of leonurine and stachydrine in normal rats and rats with Cold-Stagnation and Blood-Stasis primary dysmenorrhoea after the administration of Leonurus japonicus houtt electuary. J Sep Sci 42: 1725-1732, 2019.

40. Dan-Dan L, Hua-Sheng P, Yuan Z, Xian-Zhang H, Zhi-Lai Z Da-Hui L, Li-Ping K and Lu-Qi H: Comparison of chemical components between Artemisia stolonifera and Artemisia argyi using UPLC-Q-TOF-MS. Zhongguo Zhong Yao Za Zhi 45: 4057-4064, 2020 (In Chinese). 
41. Wang ZL, Gao HM, Wang S, Zhang M, Chen K, Zhang YQ, Wang HD, Han BY, Xu LL, Song TQ, et al: Dissection of the general two-step di-C-glycosylation pathway for the biosynthesis of (iso)schaftosides in higher plants. Proc Natl Acad Sci USA 117 30816-30823, 2020.

42. Suntornsuk L, Kasemsook S and Wongyai S: Quantitative analysis of aglycone quercetin in mulberry leaves (Morus alba L.) by capillary zone electrophoresis. Electrophoresis 24: 1236-1241, 2003.

43. Kim GD, Lee YS, Cho JY, Lee YH, Choi KJ, Lee Y, Han TH, Lee SH, Park KH and Moon JH: Comparison of the content of bioactive substances and the inhibitory effects against rat plasma oxidation of conventional and organic hot peppers (Capsicum annuum L.). J Agric Food Chem 58: 12300-12306, 2010.

44. Li S, Zhou S, Yang W and Meng D: Gastro-protective effect of edible plant Artemisia argyi in ethanol-induced rats via normalizing inflammatory responses and oxidative stress. J Ethnopharmacol 214: 207-217, 2018

45. Chuang TY, Lien CY, Tsai YC, Lin KF, Hsu CH, Wu WJ, Su LY, $\mathrm{Lu} \mathrm{CW}$ and $\mathrm{Wu} \mathrm{CH}$ : Oral treatment with the Chinese herbal supplements B307 enhances muscle endurance of ICR mice after exhaustive swimming via suppressing fatigue, oxidative stress, and inflammation. Food Sci Nutr 8: 3682-3691, 2020

46. Lien CY, Lu CW, Lin YH, Wu WJ, Hsu CH, Chuang TY, Lin KF, Chuang WC, Lee MC and Wu $\mathrm{CH}$ : Chinese herbal medicine, guilu erxian glue, as alternative medicine for adverse side effects of chemotherapy in Doxorubicin-Treated cell and mouse models. Evid Based Complement Alternat Med 2021: 5548968, 2021.

47. Kato T, Iizuka K, Takao K, Horikawa Y, Kitamura T and Takeda J: ChREBP-Knockout mice show sucrose intolerance and fructose malabsorption. Nutrients 10: 340, 2018

48. Burke SJ, Batdorf HM, Martin TM, Burk DH, Noland RC Cooley CR, Karlstad MD, Johnson WD and Collier JJ: Liquid sucrose consumption promotes obesity and impairs glucose tolerance without altering circulating insulin levels. Obesity (Silver Spring) 26: 1188-1196, 2018.

49. Chen YS, Liu HM and Lee TY: Ursodeoxycholic acid regulates hepatic energy homeostasis and white adipose tissue macrophages polarization in Leptin-Deficiency obese mice. Cells 8: 253, 2019.

50. Livak KJ and Schmittgen TD: Analysis of relative gene expression data using real-time quantitative PCR and the 2(-Delta Delta C(T)) method. Methods 25: 402-408, 2001.

51. Moylan JS, Smith JD, Chambers MA, McLoughlin TJ and Reid MB: TNF induction of atrogin-1/MAFbx mRNA depends on Foxo4 expression but not AKT-Foxo1/3 signaling. Am J Physiol Cell Physiol 295: C986-C993, 2008.

52. Qian Y, Guo X, Che L, Guan X, Wu B, Lu R, Zhu M, Pang H, Yan Y, Ni Z and $\mathrm{Gu} \mathrm{L}$ : Klotho reduces necroptosis by targeting oxidative stress involved in renal Ischemic-Reperfusion injury. Cell Physiol Biochem 45: 2268-2282, 2018.

53. Omede F, Zhang S, Johnson C, Daniel E, Zhang Y, Fields TA, Boulanger J, Liu S, Ahmed I, Umar S, et al: Dietary phosphate restriction attenuates polycystic kidney disease in mice. Am J Physiol Renal Physiol 318: F35-F42, 2020.

54. Murdoch CE, Alom-Ruiz SP, Wang M, Zhang M, Walker S, Yu B, Brewer A and Shah AM: Role of endothelial Nox 2 NADPH oxidase in angiotensin II-induced hypertension and vasomotor dysfunction. Basic Res Cardiol 106: 527-538, 2011.

55. Sag CM, Schnelle M, Zhang J, Murdoch CE, Kossmann S, Protti A, Santos CXC, Sawyer G, Zhang X, Mongue-Din H, et al Distinct regulatory effects of myeloid cell and endothelial cell NAPDH oxidase 2 on blood pressure. Circulation 135: 2163-2177, 2017.

56. Yates Z, Tarling EJ, Langley-Evans SC and Salter AM: Maternal undernutrition programmes atherosclerosis in the ApoE*3-Leiden mouse. Br J Nutr 101: 1185-1194, 2009.

57. Wang CH, Liu HM, Chang ZY, Huang TH and Lee TY: Losartan prevents hepatic steatosis and macrophage polarization by inhibiting HIF-1 $\alpha$ in a murine model of NAFLD. Int J Mol Sci 22: $7841,2021$.

58. Huang Y, Wang J, Qua G, Wang X, Yang L and Zhong L: Lactobacillus acidophilus ATCC 4356 prevents atherosclerosis via inhibition of intestinal cholesterol absorption in apolipoprotein E-knockout mice. Appl Environ Microbiol 80: 7496-7504, 2014.

59. Jin YN, Hwang WY, Jo C and Johnson GV: Metabolic state determines sensitivity to cellular stress in Huntington disease: Normalization by activation of PPAR $\gamma$. PLoS One 7: e30406, 2012
60. Shimano H, Yahagi N, Amemiya-Kudo M, Hasty AH, Osuga J, Tamura Y, Shionoiri F, Iizuka Y, Ohashi K, Harada K, et al: Sterol regulatory element-binding protein-1 as a key transcription factor for nutritional induction of lipogenic enzyme genes. J Biol Chem 274: 35832-35839, 1999.

61. Sung YY, Yoon T, Jang S and Kim HK: Forsythia suspensa suppresses house dust mite Extract-Induced atopic dermatitis in NC/Nga Mice. PLoS One 11: e0167687, 2016.

62. Saba E, Lee YS, Yang WK, Lee YY, Kim M, Woo SM, Kim K, Kwon YS, Kim TH, Kwak D, et al: Effects of a herbal formulation, KGC3P, and its individual component, nepetin, on coal fly dust-induced airway inflammation. Sci Rep 10: 14036, 2020.

63. Kagari T, Doi H and Shimozato T: The importance of IL-1 beta and TNF-alpha, and the noninvolvement of IL-6, in the development of monoclonal antibody-induced arthritis. J Immunol 169: 1459-1466, 2002

64. Choi BH, Ahn IS, Kim YH, Park JW, Lee SY, Hyun CK and Do MS: Berberine reduces the expression of adipogenic enzymes and inflammatory molecules of 3T3-L1 adipocyte. Exp Mol Med 38: 599-605, 2006.

65. Yang H, Liao D, Tong L, Zhong L and Wu K: MiR-373 exacerbates renal injury and fibrosis via NF- $\kappa \mathrm{B} /$ MatrixMetalloproteinase-9 signaling by targeting Sirtuin1. Genomics 111: 786-792, 2019.

66. Shin H, Ma Y, Chanturiya T, Cao Q, Wang Y, Kadegowda AKG, Jackson R, Rumore D, Xue B, Shi H, et al: Lipolysis in brown adipocytes is not essential for Cold-Induced thermogenesis in mice. Cell Metab 26: 764-777.e5, 2017.

67. Thornton SM, Krolopp JE and Abbott MJ: IL-15 mediates mitochondrial activity through a PPAR $\delta$-Dependent-PPAR $\alpha$-Independent mechanism in skeletal muscle cells. PPAR Res 2016: 5465804, 2016.

68. Luan G, Li G, Ma X, Jin Y, Hu N, Li J, Wang Z and Wang H: Dexamethasone-Induced mitochondrial dysfunction and insulin Resistance-Study in 3T3-L1 adipocytes and mitochondria isolated from mouse liver. Molecules 24: 1982, 2019.

69. Dusaulcy R, Rancoule C, Grès S, Wanecq E, Colom A, Guigné C, van Meeteren LA, Moolenaar WH, Valet P and Saulnier-Blache JS: Adipose-specific disruption of autotaxin enhances nutritional fattening and reduces plasma lysophosphatidic acid. J Lipid Res 52: 1247-1255, 2011.

70. Bernardi S, Toffoli B, Tisato V, Bossi F, Biffi S, Lorenzon A Zauli G, Secchiero P and Fabris B: TRAIL reduces impaired glucose tolerance and NAFLD in the high-fat diet fed mouse. Clin Sci (Lond) 132: 69-83, 2018.

71. Bermúdez-Muñoz JM, Celaya AM, Hijazo-Pechero S, Wang J, Serrano M and Varela-Nieto I: G6PD overexpression protects from oxidative stress and age-related hearing loss. Aging Cell 19: e13275, 2020

72. Wang Y, Sakata T, Elalieh HZ, Munson SJ, Burghardt A, Majumdar S, Halloran BP and Bikle DD: Gender differences in the response of CD-1 mouse bone to parathyroid hormone: Potential role of IGF-I. J Endocrinol 189: 279-287, 2006.

73. Al-Dwairi A, Brown AR, Pabona JM, Van TH, Hamdan H, Mercado CP, Quick CM, Wight PA, Simmen RC and Simmen FA: Enhanced gastrointestinal expression of cytosolic malic enzyme (ME1) induces intestinal and liver lipogenic gene expression and intestinal cell proliferation in mice. PLoS One 9: e113058, 2014.

74. Zhao L, Li C, Liu F, Zhao Y, Liu J, Hua Y, Liu J, Huang J and Ge C: A blockade of PD-L1 produced antitumor and antimetastatic effects in an orthotopic mouse pancreatic cancer model via the PI3K/Akt/mTOR signaling pathway. Onco Targets Ther 10: 2115-2126, 2017

75. Deng L, Huang L, Sun Y, Heath JM, Wu H and Chen Y: Inhibition of FOXO1/3 promotes vascular calcification. Arterioscler Thromb Vasc Biol 35: 175-183, 2015.

76. Choi DH, Yang J and Kim YS: Rapamycin suppresses postnatal muscle hypertrophy induced by myostatin-inhibition accompanied by transcriptional suppression of the Akt/mTOR pathway. Biochem Biophys Rep 17: 182-190, 2019.

77. Roselló-Díez A, Madisen L, Bastide S, Zeng H and Joyner AL: Cell-nonautonomous local and systemic responses to cell arrest enable long-bone catch-up growth in developing mice. PLoS Biol 16: e2005086, 2018.

78. Prieto C and Barrios D: RaNA-Seq: Interactive RNA-Seq analysis from FASTQ files to functional analysis. Bioinformatics: Nov 15, 2019 (Epub ahead of print).

79. Bolger AM, Lohse M and Usadel B: Trimmomatic: A flexible trimmer for Illumina sequence data. Bioinformatics 30: 2114-2120, 2014 
80. Sahraeian SME, Mohiyuddin M, Sebra R, Tilgner H, Afshar PT, Au KF, Bani Asadi N, Gerstein MB, Wong WH, Snyder MP, et al: Gaining comprehensive biological insight into the transcriptome by performing a broad-spectrum RNA-seq analysis. Nat Commun 8: 59, 2017.

81. Wang L, Wang S and Li W: RSeQC: Quality control of RNA-seq experiments. Bioinformatics 28: 2184-2185, 2012.

82. Okonechnikov K, Conesa A and García-Alcalde F: Qualimap 2: Advanced multi-sample quality control for high-throughput sequencing data. Bioinformatics 32: 292-294, 2046.

83. Liao Y, Smyth GK and Shi W: FeatureCounts: An efficient general purpose program for assigning sequence reads to genomic features. Bioinformatics 30: 923-930, 2014.

84. Varet H, Brillet-Guéguen L, Coppée JY and Dillies MA SARTools: A DESeq2- and EdgeR-Based R pipeline for comprehensive differential analysis of RNA-Seq data. PLoS One 11: e0157022, 2016.

85. Holm L and Sander C: Removing near-neighbour redundancy from large protein sequence collections. Bioinformatics 14 423-429, 1998.

86. Benjamini Y and Hochberg Y: Controlling the false discovery rate: A practical and powerful approach to multiple testing. J R Stat Soc Ser C Appl Stat 57: 289-300, 1995.

87. Liu S, Cai Y, Changyong E, Sheng J and Zhang X: Screening and validation of independent predictors of poor survival in pancreatic cancer. Pathol Oncol Res 27: 1609868, 2021.

88. Gene Ontology Consortium: The Gene Ontology project in 2008. Nucleic Acids Res 36: D440-D444, 2008

89. Zhu HM, Fei Q, Qian LX, Liu BL, He X and Yin L: Identification of key pathways and genes in nasopharyngeal carcinoma using bioinformatics analysis. Oncol Lett 17: 4683-4694, 2019.

90.Deng YJ, Ren FH, Yuan WH, Zhang GZ, Wu ZL and Xie QQ: GRB10 and E2F3 as diagnostic markers of osteoarthritis and their correlation with immune infiltration. Diagnostics (Basel) 10: 171, 2020

91. Wu T, Hu E, Xu S, Chen M, Guo P, Dai J, Feng T, Zhou L, Tang W, Zhan L, et al: clusterProfiler 4.0: A universal enrichment tool for interpreting omics data. Innovation (N Y) 2: 100141, 2021.

92.Bagul PK, Middela H, Matapally S, Padiya R, Bastia T, Madhusudana K, Reddy BR, Chakravarty S and Banerjee SK Attenuation of insulin resistance, metabolic syndrome and hepatic oxidative stress by resveratrol in fructose-fed rats. Pharmacol Res 66: 260-268, 2012.

93. Phuwamongkolwiwat P, Suzuki T, Hira $T$ and Hara H: Fructooligosaccharide augments benefits of quercetin-3-O- $\beta$-glucoside on insulin sensitivity and plasma total cholesterol with promotion of flavonoid absorption in sucrose-fed rats. Eur J Nutr 53: 457-468, 2014

94. Marzani B, Balage M, Vénien A, Astruc T, Papet I, Dardevet D and Mosoni L: Antioxidant supplementation restores defective leucine stimulation of protein synthesis in skeletal muscle from old rats. J Nutr 138: 2205-2211, 2008.

95. Slowik A, Borratynska A, Turaj W, Pera J, Dziedzic T, Figlewicz DA, Betlej M, Krzyszkowski T, Czepko R and Szczudlik A: Alpha1-antichymotrypsin gene (SERPINA3) A/T polymorphism as a risk factor for aneurysmal subarachnoid hemorrhage. Stroke 36: 737-740, 2005.

96. O'Brien M: The reciprocal relationship between inflammation and coagulation. Top Companion Anim Med 27: 46-52, 2012.

97. Levi M: The coagulant response in sepsis and inflammation. Hamostaseologie 30: 10-12, 14-16, 2010.

98. Levi $\mathrm{M}$ and van der Poll T: Inflammation and coagulation. Crit Care Med 38 (Suppl 2): S26-S34, 2010.

99. Sohn EH, Khanna A, Tucker BA, Abràmoff MD, Stone EM and Mullins RF: Structural and biochemical analyses of choroidal thickness in human donor eyes. Invest Ophthalmol Vis Sci 55: 1352-1360, 2014.

100. Nazir S, Jankowski V, Bender G, Zewinger S, Rye KA and van der Vorst EPC: Interaction between high-density lipoproteins and inflammation: Function matters more than concentration! Adv Drug Deliv Rev 159: 94-119, 2020.

101. Maïga SF, Kalopissis AD and Chabert M: Apolipoprotein A-II is a key regulatory factor of HDL metabolism as appears from studies with transgenic animals and clinical outcomes Biochimie 96: 56-66, 2014.
102. da Silva IT, de Almeida-Pititto B and Ferreira SRG: Reassessing lipid metabolism and its potentialities in the prediction of cardiovascular risk. Arch Endocrinol Metab 59: 171-180, 2015.

103. Lehman JJ, Barger PM, Kovacs A, Saffitz JE, Medeiros DM and Kelly DP: Peroxisome proliferator-activated receptor gamma coactivator-1 promotes cardiac mitochondrial biogenesis. J Clin Invest 106: 847-856, 2000.

104. Price NL, Gomes AP, Ling AJ, Duarte FV, Martin-Montalvo A, North BJ, Agarwal B, Ye L, Ramadori G, Teodoro JS, et al: SIRT1 is required for AMPK activation and the beneficial effects of resveratrol on mitochondrial function. Cell Metab 15: 675-690, 2012

105. Vazquez F, Lim JH, Chim H, Bhalla K, Girnun G, Pierce K, Clish CB, Granter SR, Widlund HR, Spiegelman BM and Puigserver P: PGC1 $\alpha$ expression defines a subset of human melanoma tumors with increased mitochondrial capacity and resistance to oxidative stress. Cancer Cell 23: 287-301, 2013.

106. Zhang L, Zhou Y, Wu W, Hou L, Chen H, Zuo B, Xiong Y and Yang J: Skeletal muscle-specific overexpression of PGC-1a induces Fiber-type conversion through enhanced mitochondrial respiration and fatty acid oxidation in mice and pigs. Int $\mathrm{J}$ Biol Sci 13: 1152-1162, 2017

107. Lin J, Wu H, Tarr PT, Zhang CY, Wu Z, Boss O, Michael LF, Puigserver $\mathrm{P}$, Isotani E, Olson EN, et al: Transcriptional co-activator PGC-1 alpha drives the formation of slow-twitch muscle fibres. Nature 418: 797-801, 2002.

108. Puigserver P, Wu Z, Park CW, Graves R, Wright $\mathrm{M}$ and Spiegelman BM: A cold-inducible coactivator of nuclear receptors linked to adaptive thermogenesis. Cell 92: 829-839, 1998.

109. Eisele PS, Salatino S, Sobek J, Hottiger MO and Handschin C: The peroxisome proliferator-activated receptor $\gamma$ coactivator $1 \alpha / \beta$ (PGC-1) coactivators repress the transcriptional activity of NF- $\kappa \mathrm{B}$ in skeletal muscle cells. J Biol Chem 288: 2246-2260, 2013.

110. Lira VA, Benton CR, Yan Z and Bonen A: PGC-1alpha regulation by exercise training and its influences on muscle function and insulin sensitivity. Am J Physiol Endocrinol Metab 299: E145-E161, 2010.

111. Sandri M, Sandri C, Gilbert A, Skurk C, Calabria E, Picard A, Walsh K, Schiaffino S, Lecker SH and Goldberg AL: Foxo transcription factors induce the atrophy-related ubiquitin ligase atrogin-1 and cause skeletal muscle atrophy. Cell 117: 399-412, 2004

112. Stitt TN, Drujan D, Clarke BA, Panaro F, Timofeyva Y, Kline WO, Gonzalez M, Yancopoulos GD and Glass DJ: The IGF-1/PI3K/Akt pathway prevents expression of muscle atrophy-induced ubiquitin ligases by inhibiting FOXO transcription factors. Mol Cell 14: 395-403, 2004.

113. Guttridge DC, Mayo MW, Madrid LV, Wang CY and Baldwin AS Jr: NF-kappaB-induced loss of MyoD messenger RNA: Possible role in muscle decay and cachexia. Science 289: 2363-2366, 2000.

114. Kang C, Shin WS, Yeo D, Lim W, Zhang T and Ji LL: Anti-inflammatory effect of avenanthramides via NF- $\kappa B$ pathways in $\mathrm{C} 2 \mathrm{C} 12$ skeletal muscle cells. Free Radic Biol Med 117: 30-36, 2018.

115. Solomon V and Goldberg AL: Importance of the ATP-ubiquitin-proteasome pathway in the degradation of soluble and myofibrillar proteins in rabbit muscle extracts. J Biol Chem 271: 26690-26697, 1996.

116. Scott SV and Klionsky DJ: Delivery of proteins and organelles to the vacuole from the cytoplasm. Curr Opin Cell Biol 10: 523-529, 1998

117. Lecker SH, Goldberg AL and Mitch WE: Protein degradation by the ubiquitin-proteasome pathway in normal and disease states. J Am Soc Nephrol 17: 1807-1819, 2006.

118. Papanicolaou KN, Izumiya Y and Walsh K: Forkhead transcription factors and cardiovascular biology. Circ Res 102: 16-31, 2008 .

This work is licensed under a Creative Commons Attribution-NonCommercial-NoDerivatives 4.0 International (CC BY-NC-ND 4.0) License. 